Final Report

on the Purchase Order B-192560

A.V.Anikeev, P.A.Bagrianskii, P.A.Deichuli,

A.A.Ivanov, A.N.Karpushov, V.N.Khudik,

I.A.Kotelnikov, I.M.Lansky, A.I.Rogozin,

- D.D.Ryutov, T.V.Salikova

The Budker Institute of Nuclear Physics

March 1993

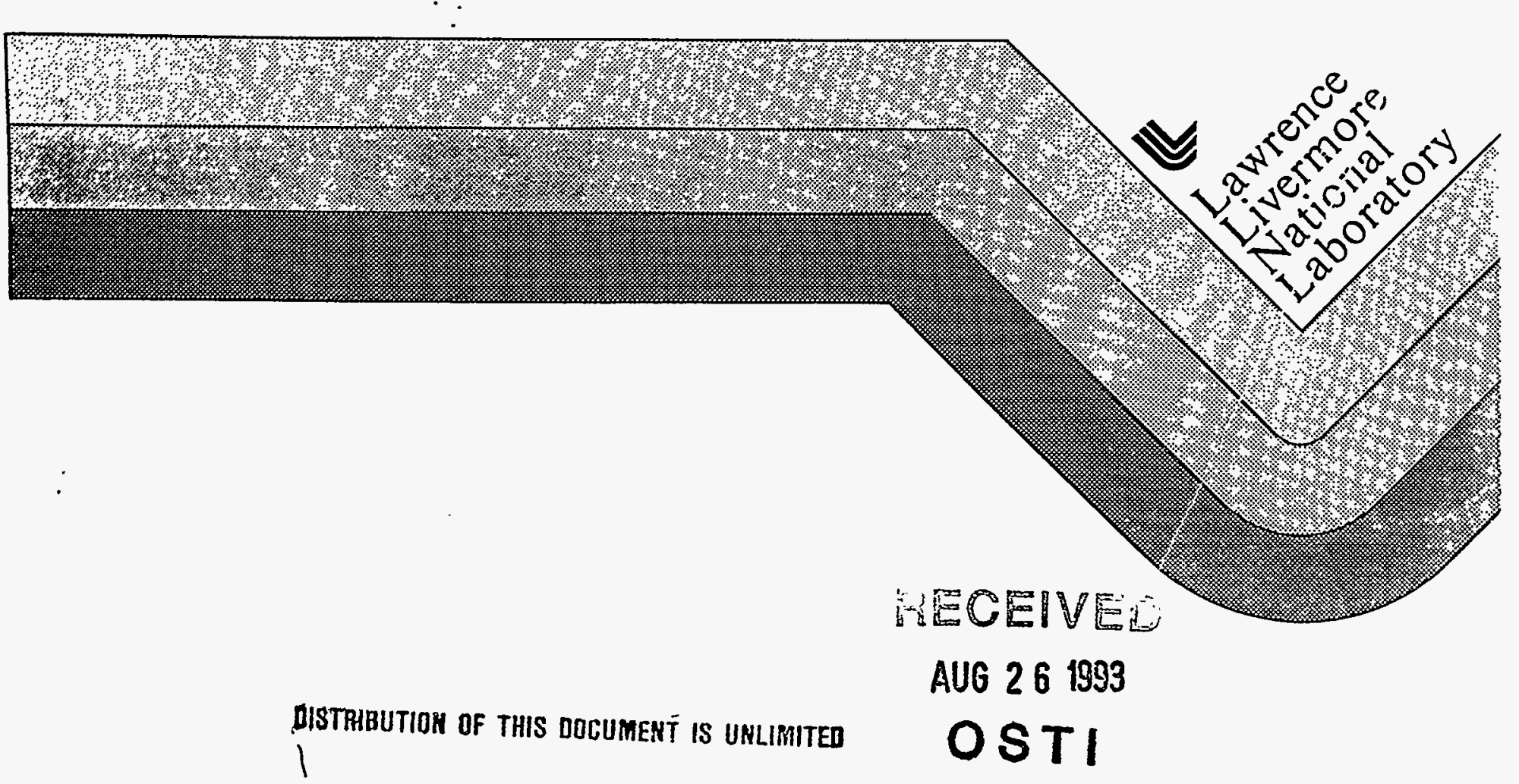




\section{DISCLAIMER}

This report was prepared as an account of work sponsored by an agency of the United States Government. Neither the United States Government nor any agency thereof, nor any of their employees, make any warranty, express or implied, or assumes any legal liability or responsibility for the accuracy, completeness, or usefulness of any information, apparatus, product, or process disclosed, or represents that its use would not infringe privately owned rights. Reference herein to any specific commercial product, process, or service by trade name, trademark, manufacturer, or otherwise does not necessarily constitute or imply its endorsement, recommendation, or favoring by the United States Government or any agency thereof. The views and opinions of authors expressed herein do not necessarily state or reflect those of the United States Government or any agency thereof. 


\section{DISCLAIMER}

Portions of this document may be illegible in electronic image products. Images are produced from the best available original document. 


\title{
FINAL REPORT \\ on the Purchase Order B-192560
}

\author{
A.V.Anikeev, P.A.Bagrianskii, P.A.Deichuli, \\ A.A.Ivanov, A.N.Karpushov, V.N.Khudik, \\ I.A.Kotelnikov, I.M.Lansky, A.I.Rogozin, \\ - D.D.Ryutov, T.V.Salikova
}

The Budker Institute of Nuclear Physics
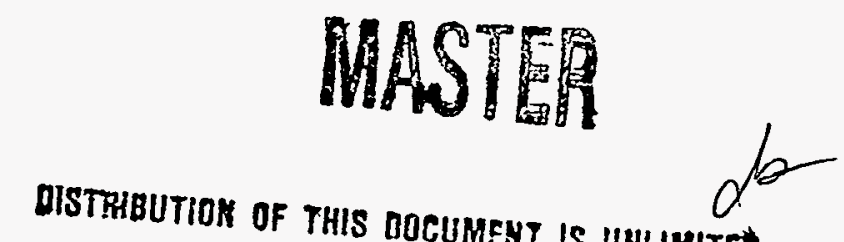


\section{ABSTRACT}

This is final report on the Purchase Order B-192560. It consists of 3 chapters.

Chapter 1 contains stability analysis of axisymmetric plasma configurations in which there exists a population of "hot" particles whose drift frequency around the magnetic axis greatly exceeds the frequency of the flute perturbation. It is shown that fast drifting particles affect not potential energy of MHD perturbations but their kinetic energy. The possibility of the stabilization of the flute instability due to the "negative inertia" effect is discussed. Results, obtained in this chapter, are of considerable importance for the stability of various systems with hot particles.

Chapter 2 is devoted to the theory of non-paraxial MHD stabilizers for axisymmetric mirror devices for plasma confinement. In this chapter, a method of search of axisymmetric configurations which provide stability of rigid $(m=1)$ flute mode is given and several theorems, elucidating the effects of non-paraxiality on flute modes stabilization by sloshing ions are proved.

Chapter 3 presents experimental part of the work. Two interrelated sets of experiments were planned for the GDT facility in order to study the problems included into an experimental part of the work. The first was intended for measurements of a pressure-weighted curvature observing a plasma equilibrium response to applying a small test (dipole in a simplest version, or may be of a higher order) perturbation of magnetic field in the MHD-anchor region. A simple magneto-hydrodynamic model is used to deduce from experimental data the pressure-weighted curvature of the field lines that determines a plasma stability against flute modes. Main assets of the approach are its simplicity and clearness, which allows it to be served as one of the mains. It is also potentially of great utility to control the displacements of the plasma from geometrical axis of the trap which arise in many cases due to uncontrolled errors in mounting of the coils, soft-iron parts near the machine, etc. Response to quadrupole external disturbances were also studied. Significant difference between measured response and its theoretical estimate was found. 
Another set of experiments was devoted to studying the stabilization of the flutes by a cusp end cell. The cusp cell was attached to the GDT device at early 1992 . The cusp coils was electrically tested and desired parameters were achieved. Within. the frame of this Purchase Order we accurately measured plasma parameters in the cusp, studied stability limits and measured some spatial characteristics of the unstable MHD-modes above the instability threshold. 


\section{Contents}

1 Stability of axisymmetric plasma containing fast drifting particles 1

1.1 Introduction . . . . . . . . . . . . . . . 1

1.2 Generalized energy principle for axisymmetric plasma configurations . 5

1.3 Stability of the "global" mode . . . . . . . . . . . . 9

1.4 "Negative inertia" stabilization in the magnetic mirror . . . . . . 12

1.5 "Negative inertia" stabilization in the cusp . . . . . . . . 15

1.6 Nonlinear stability . . . . . . . . . . . . . . . 17

1.7 Application to the beam-plasma neutron source $\ldots \ldots \ldots 21$

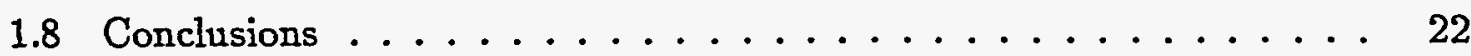

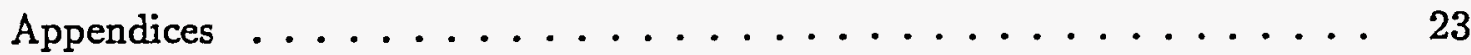

A Van Dam-Rosenbluth-Lee energy principle in the case of the flute perturbations . . . . . . . . . . . . . . 23

B Energy principle for the "disk"-like plasma . . . . . . . . . 29

C Generalization of the energy principle for the case: $\Gamma / m \ll \Omega_{d} \leq \Gamma \ldots 30$

2 Non-paraxial axisymmetric MHD anchors 35

2.1 Stability of disk-shaped plasma . . . . . . . . . . 36

2.1.1 System of two magnetic dipoles . . . . . . . . 36

2.1.2 System of two close coils . . . . . . . . . . . . 41

2.2 Stability of isotropic plasma . . . . . . . . . . . 44

2.3 Stabilization by sloshing ions . . . . . . . . . . . 45 
3 Experiments on the GDT facility

3.1 Measurements of a plasma equilibrium response to external multipole fields in an axisymmetric gas-dynamic trap . . . . . . . . 50

3.1 .1 Introduction . . . . . . . . . . . . 50

3.1.2 Estimates of the equilibrium parameters in the presence of multipole disturbances. . . . . . . . . . . . . . 54

3.1.3 Results of measurements. . . . . . . . . . . 56

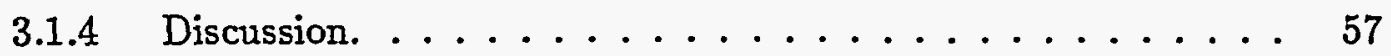

3.2 A neutral beam probe for measurements of density fluctuations in the GDT experiment. . . . . . . . . . . . . 58

3.3 Stability properties of a cusp-anchored gas-dynamic trap. . . . . . 60

3.3.1 Introduction. . . . . . . . . . . . . 60

3.3.2 Experiments with Gun-Produced Plasma. . . . . . . . . 61

3.3.3 Experiments with Neutral Beam Heating. . . . . . . . . 63 


\section{Chapter 1}

\section{Stability of axisymmetric plasma containing fast drifting particles}

\subsection{Introduction}

Axial symmetry is a very desirable property of the mirror devices both for fusion and neutron source applications. The main obstacle to be circumvented in the development of such systems, is the flute instability of axisymmetric mirrors. In recent years there appeared a number of proposals, devoted to the stabilization of the flute perturbations in the framework of axisymmetric magnetic configurations, which are based on the combining of the MHD unstable central cell with various types of end-cell stabilizers [1]-[3]. In the present Chapter we concentrate ourselves just on this scheme, including long solenoid with a uniform field, conjugated with the end stabilizing anchor, intended to provide MHD stability of the system as a whole. The attractive feature of such a configuration is that it allows to exploit finite larmor radius (FLR) effects [5] for the stabilization of the flute perturbations. As is well known [5], FLR effects, being strong, stabilize all flute modes, except the one with azimuthal number " $m=1$, corresponding to the "rigid" displacement of the plasma column (the "global" mode). Consequently, in the conditions when FLR effects dominate, the anchor has 
to stabilize the "global" mode only. Bearing in mind a favorable influence of FLR effects we, however, don't restrict our research by discussion of only "global" mode stability and consider a general case of an arbitrary azimuthal mode.

The conventional approach to the stability analysis (used, for instance, in $[1,2]$ ) contains an assumption that the curvature-induced particle drift is slow as compared with the scale-time of the flute perturbation growth,

$$
\Omega_{d} \ll \Gamma
$$

where $\Gamma$ denotes a characteristic growth-rate of the flute perturbation (or a characteristic frequency in the stable case), and $\Omega_{d}$ is a characteristic drift frequency in the stabilizing anchor. Inequality (1.1) means that plasma particles in the anchor undergo displacement remaining within the flute flux tube that they were initially occupying. Therefore their perturbed position can be defined by 2-D hydrodynamic displacement vector $\boldsymbol{\xi}$, being the same for all particles, occupying in the initial state some flux tube, irrespective to their velocity and pitch angle. So, one can analyze the stability of the system in the framework of the familiar Kruskal-Oberman energy principle [5]. Just this approach was used in [1,2].

On the other hand, the presence of a long central cell, possessing large inertia, can result in the violation of (1.1). Indeed, flute growth-rate in the central cell itself (without the anchor) scales as [8]

$$
\Gamma \sim v_{T i} / \sqrt{L_{c} L_{t r}}
$$

where $L_{c}, L_{t r}$ are lengths of solenoidal and transition regions respectively, and $v_{T i}$ is the ion thermal velocity in the central cell. It looks hardly probable to achieve a stabilizing contribution of the anchor more than 1.5-2 times exceeding the unstable contribution of the central cell. Hence, if one switches on the anchor, the characteristic . frequency of the flute oscillations keeps its former value (1.2), just the sign of $\Gamma^{2}$ changes. Drift frequency around the magnetic axis in the anchor region is estimated 
as follows:

$$
\Omega_{d} \sim \frac{c T_{s}}{e B_{s} L_{s}^{2}},
$$

$B$, being the magnetic field in the stabilizer middle plane, $T_{s}$ being plasma temper: ature in the stabilizer (to avoid unnecessary notation overloading in estimates, we suppose electron and ion temperatures in the anchor to be equal to each other). As it follows from (1.2),(1.3), growth-rate $\Gamma$ becomes really small as compared with the drift frequency when $L_{c}$ is large enough.

The latter conclusion gives rise to the interest for the investigation of the situation when the drift frequency in the stabilizing anchor exceeds essentially the frequency of MHD perturbations, i.e. when inequality inverse to (1.1) is satisfied:

$$
\Omega_{d} \gg \Gamma
$$

To analyze the stability of such a system one cannot use Kruskal-Oberman energy principle and has to rely on its modified version that takes into account the condition (1.4).

The generalized energy principle accounting for the contribution of nonhydrodynamic (in the sense of (1.4)) plasma species has been first formulated in [7], where single-particle adiabatic invariant technique has been used to calculate the perturbation of the particle energy. The energy variation, derived in [7], consists of the contributions of both perturbed plasma and magnetic energies. However, if one inserts into the expression for $W$, presented in [7], the displacement vector $\xi$ of the flute perturbation

$$
\xi=\frac{\nabla \eta \times \mathbf{B}}{B^{2}}
$$

with $\eta$ constant along the field line, then one comes to a somewhat paradoxical result. Namely; it turns out that $W$, as given in [7], becomes identically zero (see Appendix $A$ ). The reason is that authors of paper [7] retained in $W$ only terms proportional to $\xi^{2}$ while, as it has been shown in [8], the contribution of non-MHD particles to the energy of the perturbations has a different structure, being proportional to $\dot{\xi}^{2}$. 
Henceforth in this Chapter we consider just the case of purely flute perturbations (1.5).

Now it's appropriate to mark one more aspect. The stabilization due to strong FLR effects, mentioned above, takes place in the conditions [5]

$$
\Gamma \ll \frac{c T_{c}}{e B_{c} a_{c}^{2}},
$$

where $B_{c}, T_{c}, a_{c}$ are magnetic field, temperature and plasma radius in the central cell. Combining (1.1), (1.3), (1.6) and taking into account the conservation of the magnetic flux through the plasma cross-section,

$$
B_{c} a_{c}^{2} \sim B_{s} L_{s}^{2}
$$

(here we suppose that radial dimension of plasma in the stabilizer is of the same order of its axial dimension $L_{s}$ ) one can find that the assumption of the dominating FLR effects in the limit (1.1) is valid only if

$$
T_{s} \ll T_{c}
$$

Since the latter condition is rather restrictive, it stresses all the more the actuality of the stability analysis, involving particles with fast drift frequency (1.4).

The contents of the present chapter is as follows. In the next Section we reproduce the derivation of the generalized energy principle [8] for the axisymmetric case. In Section 1.3 we invoke the cited energy principle to illustrate the possibility of the stabilization of the flute instability due to the "negative inertia" effect. The investigation of the conditions, required for the "negative inertia" stabilization in the magnetic mirror and cusp, is the subject of Section 1.4 and Section 1.5, respectively. The influence of the nonlinear effects on the stability of plasma with fast drifting particles is examined in Section 1.6. Section 1.7 is devoted to the application of the generalized energy principle to the stability analysis of the neutron source device, proposed in [9]. Section 1.8 contains conclusions. Several computational subjects are carried out in Appendices. In particular, in Appendix $\mathrm{C}$ we show that the energy principle [8] 
admits the generalization to the intermediate frequency interval, $\Gamma / m \ll \Omega_{d} \leq \Gamma$, for modes with high azimuthal numbers $m$.

\subsection{Generalized energy principle for axisymmet- ric plasma configurations}

Suppose that in the anchor region there exists a population of hot particles with drift frequencies satisfying inequality (1.4), while other plasma both in the anchor and in the central cell is cold enough to admit conventional MHD treatment. We examine the stability of such a system with respect to the purely electrostatic perturbations that are characterized by the electrostatic potential $\varphi$, constant along the field line. The latter assumption, identical to the one made in [7], implies the presence of a cold plasma component which justifies the constancy of the potential along the field line and acts to provide quasineutrality condition.

It is convenient to adopt the following coordinate system: we mark every field line with the polar coordinates $r, \psi$ of its intersection with some plane, perpendicular to the uniform magnetic field in the central solenoid. Instead of $r$ one can use the magnetic flux $\Phi$ inside the cylindrical surface of the radius $r: \Phi=\pi r^{2} B_{c}$. A drift surface can then be described by the equation $\Phi=\Phi(\psi)$. In the introduced coordinates potential $\varphi$ of the perturbations, corresponding to the mode with azimuthal number $m$, is given by

$$
\varphi=\bar{\varphi}(\Phi) \cos m \psi
$$

Note that

$$
\bar{\varphi}(0)=0,
$$

since there is no azimuthal component of the electric field on the magnetic axis.

At a given configuration of magnetic and electrostatic fields, the drift surface for a particle with a total energy $\varepsilon$ and magnetic moment $\mu$ is determined by the constancy 
of the longitudinal action

$$
J(\varepsilon, \mu, \Phi, \psi)=(2 M)^{1 / 2} \int(\varepsilon-\mu B-e \varphi)^{1 / 2} d l
$$

with the integration carried out between the turning points. If the condition (1.4) is satisfied, then, with the electrostatic potential varying, the drift surface adjusts itself to keep constant the magnetic flux inside the surface [10]. This occurs via variation of the particle energy.

To find the change $W$ of the kinetic energy of the particles (just this quantity enters the energy principle for the perturbations with a scale-length much in excess of the Debye radius), we use the following approach. We consider some group of particles (of a total number $\Delta N$ ) that in the initial state have certain values of $\varepsilon$ and $\mu$, and that are filling a drift surface characterized with a certain value of $J$. When we slowly turn on the electrostatic potential of the perturbation, the drift surface deforms and the kinetic energy of the particles changes. If we find the change of the kinetic energy $\Delta W$ for this group, then, by summation over all the groups, we find the required quantity $W$.

The group $\Delta N$ is drifting along the contour $\Phi(\psi)$ determined by the instantaneous configuration of the electrostatic field and the instantaneous value of $\varepsilon$. The number of particles from this group $d \Delta N$, occupying the section of the contour of the arc length $d \psi$, can be presented in the form $d \Delta N=\nu d \psi$, where $\nu$ is the number of particles per unit arc length. The stationarity condition $\nu \dot{\psi}=$ const yields:

$$
\nu=\frac{\Delta N \Omega_{d}}{2 \pi \dot{\psi}}
$$

where $\dot{\psi}$ is the angular velocity of the bounce-averaged drift motion [11]:

$$
\dot{\psi}=-\frac{2 \pi c}{e t_{\|}} J_{\Phi},
$$

$t_{\|}=J_{e}$ is the transit time between the turning points, and $\Omega_{d}$ is the drift frequency,

$$
\Omega_{d}=2 \pi\left[\int_{0}^{2 \pi} \frac{d \psi}{\dot{\psi}}\right]^{-1}
$$


We use notations $J_{\varepsilon} \equiv \partial J / \partial \varepsilon, J_{\Phi} \equiv \partial J / \partial \Phi$, etc., for the partial derivatives. The change of the kinetic energy of these particles is, obviously,

$$
\Delta W=\left.\left[\int_{0}^{2 \pi} \nu(\varepsilon-e \varphi) d \psi\right]\right|_{\text {initial }} ^{\text {final }},
$$

where the subscripts indicate the difference between the final and initial state. The total energy $\varepsilon$ of particles is constant over the drift surface in the time-scale of $\Omega_{d}^{-1}$, while their kinetic energy $\varepsilon-e \varphi$ varies from one field line to another according to variation of $\varphi$. In this respect, $\Delta W$, if divided by $\Delta N$, represents the average (over the drift surface) change of the kinetic energy.

The condition of flux conservation inside the drift surface can be written in the form:

$$
\left.\int_{0}^{2 \pi} \Phi(\psi) d \psi\right|_{\text {initial }} ^{\text {final }}=0
$$

where $\Phi(\psi)$ is a solution of the equation

$$
\left.J(\varepsilon, \mu, \Phi, \psi)\right|_{\text {initial }} ^{\text {finl }}=0 .
$$

In principle, equations (1.11)-(1.16) allow one to find the particle kinetic energy at arbitrarily large $\varphi$. However, we will consider only the case of small $\varphi$. The quantities of the first order in $\varphi$ will be denoted with subscript " 1 ", the second order corrections by subscript "2", etc.

In the linear approximation, equation (1.16) yields:

$$
\left(\varepsilon_{1}-e \varphi\right) J_{\epsilon}+\Phi_{1} J_{\Phi}=0
$$

wherefrom, taking into account relationships (1.8) and (1.15), we find that

$$
\varepsilon_{1}=0, \quad \Phi_{1}=e \varphi \frac{J_{e}}{J_{\Phi}}
$$

The next order expansion of (1.16) gives:

$$
J_{\varepsilon}\left(\varepsilon_{2}-e \Phi_{1} \varphi_{\Phi}\right)+J_{\Phi} \Phi_{2}+\frac{1}{2} J_{e \varepsilon}(e \varphi)^{2}-J_{e \Phi} \Phi_{1} e \varphi+\frac{1}{2} J_{\Phi \Phi} \Phi_{1}^{2}=0
$$


The requirement (1.15), when applied to $\Phi_{2}$, yields:

$$
\int_{0}^{2 \pi} d \psi\left(\varepsilon_{2}-e \Phi_{1} \varphi_{\Phi}\right)=-\int_{0}^{2 \pi} \frac{d \psi}{J_{\varepsilon}}\left(\frac{1}{2} J_{e \varepsilon} \varphi^{2}-J_{e \Phi} \Phi_{1} e \varphi+\frac{1}{2} J_{\Phi \Phi} \Phi_{1}{ }^{2}\right)
$$

while from (1.14) we find that

$$
\Delta W=\frac{\Delta N}{2 \pi} \int_{0}^{2 \pi}\left[\frac{\dot{\psi}_{1}}{\dot{\psi}} e \varphi+\left(\varepsilon_{2}-e \Phi_{1} \varphi_{\Phi}\right)\right] d \psi
$$

From equation (1.12) one obtains that

$$
\frac{\dot{\psi}_{1}}{\dot{\psi}}=-\frac{J_{\epsilon}}{J_{\Phi}} e \varphi_{\Phi}+\frac{J_{e}}{J_{\Phi}}\left(\frac{J_{\Phi \Phi}}{J_{\Phi}}-\frac{2 J_{e \Phi}}{J_{\epsilon}}+\frac{J_{\varepsilon e} J_{\Phi}}{J_{\varepsilon}^{2}}\right) \epsilon \dot{\varphi} .
$$

Now, using relationships (1.8), (1.18), (1.21) and (1.22), we can express $\Delta W$ in terms of $\dot{\varphi}$ :

$$
\Delta W=-\frac{1}{4} e^{2} \Delta N\left[\frac{J_{\varepsilon}}{J_{\Phi}} \frac{\partial \tilde{\varphi}^{2}}{\partial \Phi}+\tilde{\varphi}^{2}\left(2 \frac{J_{e \Phi}}{J_{\Phi}}-\frac{J_{e e}}{J_{e}}-\frac{J_{e} J_{\Phi \Phi}}{J_{\Phi}^{2}}\right)\right]
$$

To perform the summation over the plasma particles, we introduce the distribution function $F(\varepsilon, \mu, \Phi)$, normalized according to the relationship $\Delta N=F(\varepsilon, \mu, \Phi) \Delta \varepsilon \Delta \mu \Delta \Phi$. Then, the energy $W$ of the perturbation acquires its final form:

$$
\begin{aligned}
W=-\frac{1}{4} e^{2} \int d \varepsilon d \mu d \Phi F(\varepsilon, \mu & , \Phi) \times \\
& \times\left[\frac{J_{\varepsilon}}{J_{\Phi}} \frac{\partial \tilde{\varphi}^{2}}{\partial \Phi}+\tilde{\varphi}^{2}\left(2 \frac{J_{\varepsilon \Phi}}{J_{\Phi}}-\frac{J_{\varepsilon \varepsilon}}{J_{\varepsilon}}-\frac{J_{\varepsilon} J_{\Phi \Phi}}{J_{\Phi}^{2}}\right)\right] .
\end{aligned}
$$

Sometimes it's more convenient to use the expression for $W$ in the form

$$
\begin{aligned}
W=\frac{1}{4} \epsilon^{2} \int d \varepsilon d \mu d \Phi \tilde{\varphi}^{2} \times \\
\times\left[\frac{\partial}{\partial \Phi}\left(F \frac{J_{\epsilon}}{J_{\Phi}}\right)-F\left(2 \frac{J_{\varepsilon \Phi}}{J_{\Phi}}-\frac{J_{e \varepsilon}}{J_{e}}-\frac{J_{e} J_{\Phi \Phi}}{J_{\Phi}^{2}}\right)\right],
\end{aligned}
$$

which can be obtained from (1.24) through the integration by parts of the first term in the square brackets (1.24). There is no contribution of limits from the integration by parts in (1.25), since on the upper limit there are no particles, and so the distribution function equals to zero, while on the lower limit the potential $\varphi$ vanishes according to (1.9). 
In Appendix $B$ we derive also the expression for the energy variation, relevant to the conditions when the longitudinal energy of the particles is small as compared with their transverse energy. In such a case plasma is located near the point of the minimum field strength on the given field line (the so-called "disk"-like plasma), and the contribution of the particles with fast drift can be expressed as

$$
W=\frac{e^{2}}{4} \int d \mu d \Phi \frac{F}{\mu} \frac{d}{d \Phi}\left(\frac{\bar{\varphi}^{2}}{B_{0 \Phi}}\right)
$$

or after the integration by parts

$$
W=-\frac{e^{2}}{4} \int d \mu d \Phi \frac{\tilde{\varphi}^{2}}{\mu B_{0 \Phi}} \frac{\partial F}{\partial \Phi}
$$

where $B_{0}$ is the minimum value of the field strength on the magnetic surface, corresponding to the flux $\Phi$, and $B_{0 \Phi} \equiv \partial B_{0} / \partial \Phi$.

\subsection{Stability of the "global" mode}

Formulae (1.24), (1.25) define the contribution of only fast drifting particles (1.4) to the total energy variation, while there exist two more terms, originated from plasma particles with small drift frequencies (1.1), whose contribution can be obtained in the framework of MHD approach. The first one scales as

$$
W^{(k)} \cong M_{i} n_{c} a^{2} L_{c} \dot{\xi}^{2}
$$

Henceforward in this Section we consider the case of the "global" mode perturbations with $\xi$ as a displacement of the plasma column as the whole in the central cell. Expression (1.28) describes the kinetic energy of the perturbations. It is caused by inertia of ions in the long central cell.

The second term,

$$
W^{(p)} \cong-\frac{n_{c} T_{c} a^{2}}{L_{t r}} \xi^{2}
$$

describes the potential energy of the perturbations caused by the field line curvature. The sign of $W^{(p)}$ is negative due to the unfavorable field line curvature in the transition 
region. As it follows from (1.28), (1.29), in the central cell itself (with the anchor being switched off) the instability grows up with the characteristic increment given by (1.2).

Taking into consideration the contribution (1.25) of the fast particles, notice that the expression for $W$ scales as $\varphi^{2}$. Since the displacement $\xi$ of the flux tube, filled with a cold plasma, is determined by formula (1.5) with $\eta=-(1 / c) \int \varphi d t$, we see that, if (1.25) is expressed in terms of $\xi$, it scales as

$$
W \cong \frac{n_{s} L_{s}^{3}}{T_{s}} \frac{e^{2} a^{2} B_{c}^{2}}{c^{2}} \dot{\xi}^{2}
$$

Here we have supposed the radial dimension of plasma in the stabilizer to be of the order of $L_{s}$, and also condition (1.7) has been taken into account. Thus $W$ scales as a kinetic energy of the perturbations $\left(\sim \dot{\xi}^{2}\right)$, giving contribution to $W^{(k)}$, not to $W^{(p)}$. Therefore the presence of the fast drifting particles manifests itself in changing of the "inertia" of the perturbations, not of their "rigidity".

Fast drifting particles may affect the stability in two different ways, depending on the sign of the energy $W$. Positive value of $W$ gives rise to the increasing of the effective kinetic energy of the perturbations (or of their effective "inertia") that, in turn, leads to the decreasing of the increment $\Gamma$, but the instability still remains.

It seems to be more attractive another situation, when $W$ is negative and, moreover, the following requirement is satisfied:

$$
W^{(k)}+W<0 .
$$

The negative sign of the effective kinetic energy corresponds to the oscillations of the perturbations with the "negative inertia" in a hump-like potential (1.29). Since the frequency of such oscillations is, obviously, real, condition (1.31) allows one to conclude that the system becomes stable.

Estimates (1.28), (1.30), together with (1.31), impose one more constraint on the plasma parameters:

$$
\frac{n_{s}}{n_{c}} \frac{L_{s}}{L_{c}}>\frac{\rho_{H s}^{2}}{L_{s}^{2}} \frac{B_{s}^{2}}{B_{c}^{2}},
$$


where $\rho_{H s}$ is the ion larmor radius of the hot particle in the stabilizer region.

To cite one more example of the magnetic configuration in which the fast drifting particles may considerably affect the stability of the plasma we consider a single nonparaxial mirror of length $L$ (with a plasma occupying a volume of the order of $L^{3}$ ). Let plasma consist of a thermal population with temperature $T$ and density $n$, and a hot population with temperature $T_{*}$ and density $n_{*}<n$; let also the pressure of the hot component exceed that of the cold one: $n_{*} T_{*}>n T$. For the mode of a "global" displacement one can evaluate the plasma kinetic energy (per unit volume) as

$$
\left(\frac{n_{*} T_{*}}{\Omega_{d}^{2} L^{2}}+n M\right) \dot{\xi}^{2}
$$

where $\xi$ is a (small) plasma displacement. The first term here represents a contribution of the fast particles. The potential energy is just

$$
n T(\xi / L)^{2},
$$

as fast particles do not contribute to it. If the drift frequency of the fast particles $\Omega_{d}$ is not too high,

$$
\Omega_{d}<\frac{1}{L}\left(\frac{n_{*} T_{*}}{n M}\right)^{1 / 2},
$$

the inertia of the fast particles dominates. The estimate for the growth-rate $\Gamma$ is then

$$
\Gamma \sim \Omega_{d}\left(\frac{n T}{n_{*} T_{*}}\right)^{1 / 2}
$$

As $n_{*} T_{*}>n T$ the growth-rate is automatically less than the drift frequency, ensuring the applicability of our analysis. So, we see that, indeed, the "inertia" of the fast drifting particles can be dominant, despite their small density.

Notice that though in the present Section we have concerned ourselves with the stability analysis of the "global" mode (estimates (1.28), (1.29), (1.32),(1.33) hold just for the "global" mode perturbations), the stabilization due to the "negative inertia" effect, discussed above, makes it possible to suppress, in principle, the instability of an arbitrary azimuthal mode. However, as the "inertia" of the oscillations is negative (see (1.31)), the dissipative instabilities are possible. 


\section{4 "Negative inertia" stabilization in the mag- netic mirror}

As it was established in Section 1.3, the necessary condition for the stabilization of the flute instability due to the "negative inertia" effect is the negative definition of the energy variation $W$ of the fast particles:

$$
W<0 \text {. }
$$

In the given magnetic field the latter inequality imposes certain restrictions on the possible profiles of the distribution function $F$ of the fast particles. In the present Section we investigate the requirements, which the distribution function $F$ should fit to satisfy (1.34) in the magnetic mirror configuration.

We start with the stability analysis of the paraxial magnetic mirror. In the framework of the paraxial approach, the plasma radial dimension should be small compared to the mirror-to-mirror distance. If we use the coordinate frame with the axis coinciding with the magnetic axis, the longitudinal invariant $J$, up to the terms linear in $\Phi$, can be written as [7]

$$
J=J^{(0)}(\varepsilon, \mu)+\Phi J^{(1)}(\varepsilon, \mu) .
$$

The neglect of the higher order terms in $\Phi$ is justified in the paraxial region. Since the variation of $F$ in $\Phi$ has a small scale-length, the first term in (1.25) is dominant, and so expression for $W$ reduces to a simplified form:

$$
W=\frac{1}{2} e^{2} \int d \varepsilon d \mu d \Phi \frac{J_{\epsilon}^{(0)}}{J^{(1)}} \bar{\varphi}^{2} \frac{\partial F}{\partial \Phi} .
$$

Since the derivative $\partial F / \partial \Phi$ defines the sign of the diamagnetic frequency, one can conclude from (1.12), (1.35), taking into account $J_{e}^{(0)}>0$, that the energy variation would be negative for those particles whose directions of the curvature-induced and diamagnetic drifts coincide, and would be positive in the opposite case.

As an example of the stability analysis of the concrete plasma configuration, we examine the stability of the hot "disk"-like plasma located in the middle plane of 
the magnetic mirror. The energy variation of such a plasma is given by formulae (1.26), (1.27). The paraxial expansion of the magnetic field in the middle plane can be presented in the form [7]:

$$
B_{0}=B-\frac{1}{4 \pi} \frac{\mathcal{B}^{\prime \prime}}{\mathcal{B}} \Phi
$$

where $B$ is the field strength on the axis and prime denotes the derivative along the axis. Note that $B^{\prime \prime}>0$, since the magnetic field has a minimum in the middle plane. Inserting (1.36) into (1.27), one obtains:

$$
W=\pi e^{2} \frac{\mathcal{B}}{\mathcal{B}^{\prime \prime}} \int d \mu d \Phi \frac{\bar{\varphi}^{2}}{\mu} \frac{\partial F}{\partial \Phi} .
$$

As it follows from the latter expression, condition (1.34), necessary for the stabilization, is satisfied for a descending plasma profile,

$$
\frac{\partial F}{\partial \Phi}<0
$$

Now we investigate in more detail the stability of the "global" mode for the discussed above plasma configuration. The potential $\varphi$, corresponding the "global" mode perturbations, is given by

$$
\varphi=\varphi_{0} \sqrt{\Phi} \cos \psi
$$

After the substitution of (1.36), (1.38) into (1.26), one can find

$$
W=-\pi e^{2} \frac{B}{\mathcal{B}^{\prime \prime}} \int d \mu d \Phi \frac{F}{\mu} .
$$

Inequality (1.34) is satisfied in this case, and so the "negative inertia" stabilization is realizable.

Up till now in the present Section the discussion has been restricted by the framework of the paraxial plasma configurations. To illustrate the influence of nonparaxiality on the stability of the plasma with fast particles, we turn to the stability analysis of the hot "disk"-like plasma, localized in the middle plane of the magnetic 
Figure 1.1: Field lines of the mirror composed of two magnetic dipoles. Bold line corresponds to the separatrix, passing through the point of zero field strength.

mirror composed of two equal co-axis magnetic dipoles (Fig. 1.1). The magnetic field in the middle plane of such a mirror can be written in the form

$$
B_{0}=B_{*} \frac{(2-x)}{2(x+1)^{5 / 2}}
$$

where $x=4 r^{2} / L^{2}, r$ is a radius in the middle plane, $L$ is a distance between dipoles and $B_{-}$is the field strength in the middle point between them. The magnetic flux can be expressed in terms of $x$ as follows:

$$
\Phi=\Phi_{s} \frac{3^{3 / 2}}{2} \frac{x}{(1+x)^{3 / 2}}
$$

where $\Phi_{s}$ denotes the flux, corresponding to the separatrix that bounds the region of the adiabatic confinement (see Fig. 1.1).

Now we examine the sign definition of the energy variation $W$ for the "global" mode perturbation (1.38). Consider the plasma envelope, involving particles located 
on the distance $R_{0}$ from the axis in the equilibrium state. Inserting (1.38), (1.40), (1.41) into (1.26), after elementary analysis one can obtain, that the energy variation $W$ occurs to be negative for the plasma envelopes with radii

$$
\frac{R_{0}}{R_{s}}<0.72
$$

where $R_{s}$ is the separatrix radius, while for radii $R_{0}$, being outside the interval (1.42), the energy $W$ has a positive sign. Hence, non-paraxial effects lead to the violation of the condition (1.34), and so the region of distant radii pays unfavorable contribution to $W$ in the sense of the possibility of the "negative inertia" stabilization.

\section{5 "Negative inertia" stabilization in the cusp}

We start with the reproducing of some basic formulae, characterized cusp magnetic configuration. In the cylindrical coordinate system with the axis of the device as $z$-axis, cusp magnetic field can be written in the form:

$$
B_{z}=2 G z, \quad B_{r}=-G r
$$

with $G$ being constant, defining the value of the magnetic field. It's convenient to present equation, that governs the magnetic field line, as follows:

$$
r^{2} z=l^{3}
$$

where parameter $l$ marks the given field line. The minimum value of the field strength on the field line $l$ is expressed as

$$
B_{0}(l)=\sqrt{3} G l
$$

Magnetic flux $\Phi$ is connected with parameter $l$ of the field lines, forming the given flux surface, through the formula

$$
\Phi=2 \pi G l^{3} .
$$


Now we proceed to the establishing of the conditions which provide the negative definition of the energy variation $W$ of the fast particles. We examine two limits:

$$
\begin{gathered}
\varepsilon-\mu B_{0} \ll \mu B_{0}, \\
\varepsilon \gg \mu B_{0} .
\end{gathered}
$$

Inequality (1.47) implies that the longitudinal energy of the particles is small as compared with their transverse energy. These particles perform small bounce oscillations nearby the point of the minimum field strength value (the "disk"-like plasma), and their energy variation $W$ is described by expressions (1.26), (1.27). The second condition, on the contrary, corresponds to the large longitudinal energy (the limiting case of the inclined injection).

Analysis of the first limit is especially trivial. Indeed, as it follows from (1.44),(1.45), the derivative $\partial B_{0} / \partial \Phi$ is positive. Hence, according to (1.27), the negative contribution to $W$ make those particles for which

$$
\frac{\partial F}{\partial \Phi}>0 \text {. }
$$

Note, that the latter condition is opposite to the one (1.37), obtained for magnetic mirror configuration in the same limit (for the "disk"-like plasma).

Inserting (1.45), (1.46), (1.38) into (1.26), we find that for the global mode perturbations the energy $W$ occurs to be positive. Consequently, one can't stabilize the global mode by the "negative inertia" effect in the conditions (1.47).

Now we examine the limit (1.48). Using (1.43), (1.44), one can derive the following expression for longitudinal action $J$ :

$$
J=J_{0} \int_{r_{1}}^{r_{2}} d \tilde{r}\left(1 \div 1 / \tilde{r}^{6}\right)^{1 / 2}\left[r_{0}-\tilde{r}\left(1+1 / \tilde{r}^{6}\right)^{1 / 2}\right]^{1 / 2},
$$

where $J_{0}=2 \sqrt{M \mu G l^{2}}, \quad r_{0}=\varepsilon / 2^{1 / 3} \mu G l$ and integration being carried between the roots of expression in the square brackets. Inequality (1.48) leads to the requirement $r_{0} \gg 1$, which, in turn, allows one to calculate approximate values of $r_{1}$ and $r_{2}$ :

$$
r_{1} \simeq 1 / \sqrt{r_{0}}, \quad r_{2} \simeq r_{0},
$$


and then to compute $J$ :

$$
J=\frac{2}{3} J_{0} r_{0}^{3 / 2}
$$

Differentiation of (1.50) with respect to $\varepsilon$ and $\Phi$, and substitution of the corresponding derivatives into (1.25) give:

$$
W=\frac{9 \pi e^{2}}{2} \int d \varepsilon d \mu d \Phi \frac{\Phi \tilde{\varphi}^{2}}{\varepsilon}\left(-\frac{\partial F}{\partial \Phi}+\frac{31}{18} \frac{F}{\Phi}\right) .
$$

Hence, one comes to the following relation

$$
\frac{\partial F}{\partial \Phi}>\frac{31}{18} \frac{F}{\Phi}
$$

that provides the negative contribution of the particles to the energy $W$.

For the potential (1.38) of the global mode perturbation, the calculation of the expression (1.24) leads, with account for (1.46), (1.50), to the positive value of $W$. The latter states that in the limit (1.48) the "negative inertia" stabilization of the global mode is impracticable.

\subsection{Nonlinear stability}

Up to this Section we have investigated the stability of plasma, containing fast drifting particles, in the linear approximation within the assumption of the infinitesimal amplitude $\varphi_{0}$ of the perturbed potential. In this approach the energy variation $W$ of the fast particles was found to be quadratic in $\varphi_{0}$ (see (1.25)). As $\varphi_{0}$ grows, the character of the relationship between $W$ and $\varphi_{0}$ modifies, since the nonlinear effects switch on. The latter may lead to the changing of the sign of $W$ and, hence, may affect the stability of the system as the whole. Therefore it is of the certain interest to calculate the energy $W$ for the finite amplitudes $\varphi_{0}$ as well. As an example of such calculations we shall carry out the nonlinear stability analysis of the hot "disk"-like plasma, localized in the middle plane of the magnetic mirror, compose of two equal co-axis magnetic dipoles (1.1). Suppose that particle energy is high enough to satisfy 
condition (1.4). Henceforward we shall concentrate ourselves on the stability of the "global" mode with the perturbation of the potential given by

$$
\varphi=\varphi_{0} \sqrt{\frac{\Phi}{\Phi_{s}}} \cos \psi
$$

Here $\Phi_{s}$ represents the magnetic flux corresponding to the separatrix, that bounds the region of the adiabatic confinement (see Fig. 1.1).

Consider a plasma envelope formed by $N$ particles, located on the magnetic surface $\Phi_{0}$ in the equilibrium state. The energy and the magnetic moment of each particle are $\varepsilon$ and $\mu$ respectively. According to (1.11)-(1.14), the energy variation of such particles can be expressed as

$$
W=N\left(\Delta \varepsilon-\frac{\Omega_{d}}{2 \pi} \int_{0}^{2 \pi} \frac{d \psi}{\dot{\psi}} e \varphi\right),
$$

where $\Delta \varepsilon$ is the changing of the total energy of the particle, constant along the drift surface. Another designations are the same as in Section 1.2. It should be stressed that formula (1.54) is valid for an arbitrary magnitude of the amplitude of the perturbed potential (not only for the infinitesimal one). Just the calculation of the function $\mathrm{IF}^{\top}\left(\varphi_{0}\right)$ is the goal of the present Section.

In the case of the "disk"-like plasma the particle transverse energy greatly exceeds its longitudinal energy, and so the kinetic energy of the particle approximately equals to $\mu B_{0}$, with the magnetic field in the mirror equatorial plane $B_{0}$, being determined by (1.40). In the same fashion as in Section 1.2, the perturbed drift surface can be described by the function $\Phi(\psi)$, which is as yet unknown. The kinetic energy of the particle on the $\Phi(\psi)$ surface is given by equation

$$
\mu B_{0}(\Phi)=\mu B_{0}\left(\Phi_{0}\right)+\Delta \varepsilon-e \varphi_{0} \sqrt{\frac{\Phi}{\Phi_{s}}} \cos \psi,
$$

where the flux $\Phi$ being determined by (1.41). Equation (1.55) allows one to define the shape of drift surface $\Phi(\psi)$ as well as to find the energy $\Delta \varepsilon$. Indeed, every value $\Delta \varepsilon$ governs some solution $\Phi(\psi)$ of equation (1.55). However, among all this solutions we have to chose the only one, that satisfies the additional requirement (1.15). Then 
the corresponding value $\Delta \varepsilon$ will be just the desired particle total energy variation. Since the presented above scheme of the solution of equation (1.55) doesn't admit the analytical approach, the problem was investigated numerically.

Once the dependence $\Phi(\psi)$ has been found, it would not be difficult to compute the second term in the right side of (1.54). For the "disk"-like plasma the angular velocity (1.12) reduces to the form

$$
\dot{\psi}=\left.\left(\mu \frac{\partial B_{0}}{\partial \Phi}+e \frac{\partial \varphi}{\partial \Phi}\right)\right|_{\Phi=\Phi(\psi)},
$$

which has a clear interpretation: the angular velocity is determined by the gradient and $E \times B$ drifts. Finally, inserting (1.56) into (1.54), one obtains the value for the energy variation $W$.

The numerical computation results are presented on Fig (1.2), where the dependence of $W / N$ versus $\varphi_{0}$ being shown. Plasma envelopes with various radii $R_{0}$ in the equilibrium state were examined. The energy $W / N$ (being equal to the variation of the kinetic energy of a single particle, averaged over the drift surface) is measured in the units of $\varepsilon$, while amplitude $\varphi_{0}$ is measured in the units of $\varepsilon / e$. The radius $R_{0}$ is normalized on the separatrix radius. Dotted lines relate to the parabolic profiles of the linear approximation.

It should be noted one more point now. Equation (1.55) possesses the solution $\Phi(\psi)$, satisfying the requirement (1.15), not for an arbitrary large $\varphi_{0}$, but for the amplitudes, less than some fixed value, determined by the radius $R_{0}$ of the envelope in the equilibrium state. The latter is a consequence of the fact that for sufficiently large $\varphi_{0}$ the drift surface breaks up, i.e. it ceases to be a closed surface. Just for this reason the curves on Fig. 1.2 terminate at various values of the maximum allowable amplitude $\varphi_{0}$.

The curves on Fig. 1.2(a)-(c) relate to the values $R_{0}<0.72 R_{s}$, that is the interval where, according to (1.42), the linear theory predicts the negative definition of the energy $W$, that, in turn, implies the possibility of the "negative inertia" stabilization. For the large amplitudes $\varphi_{0}$ the quadratic fall of the energy W slows down and then 


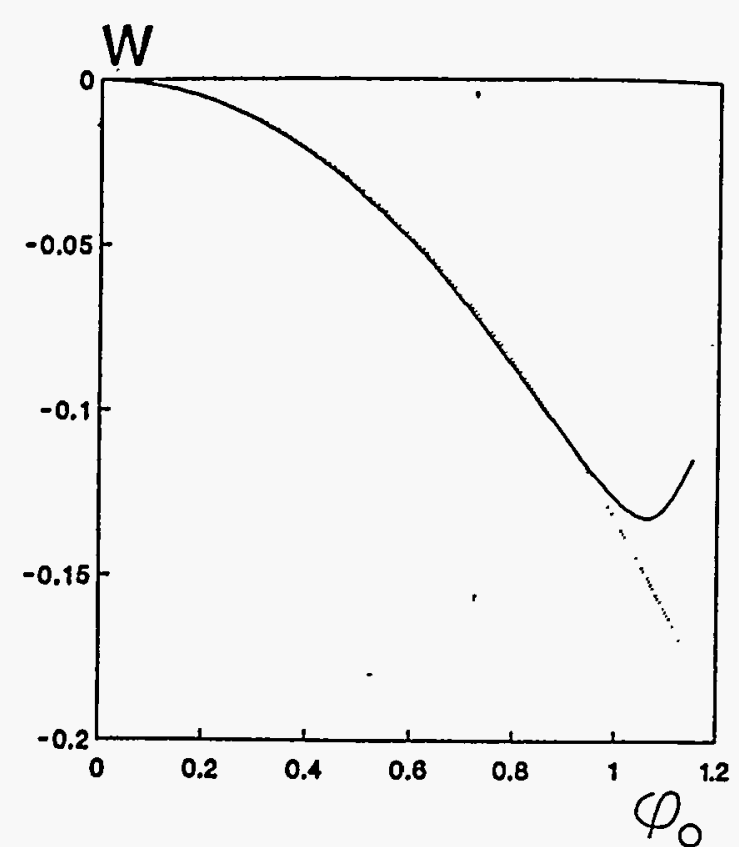

(c)

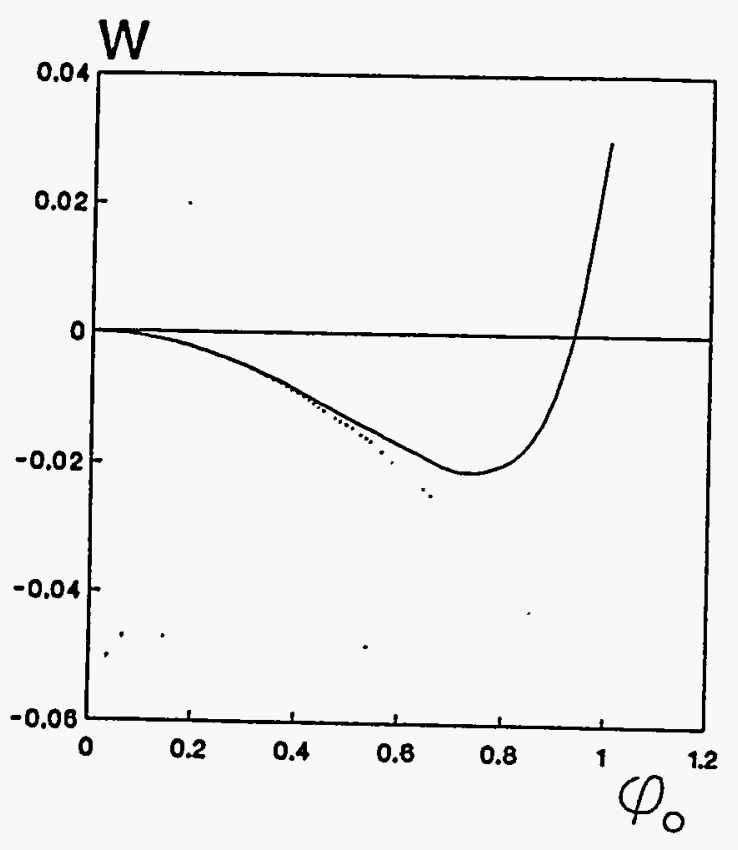

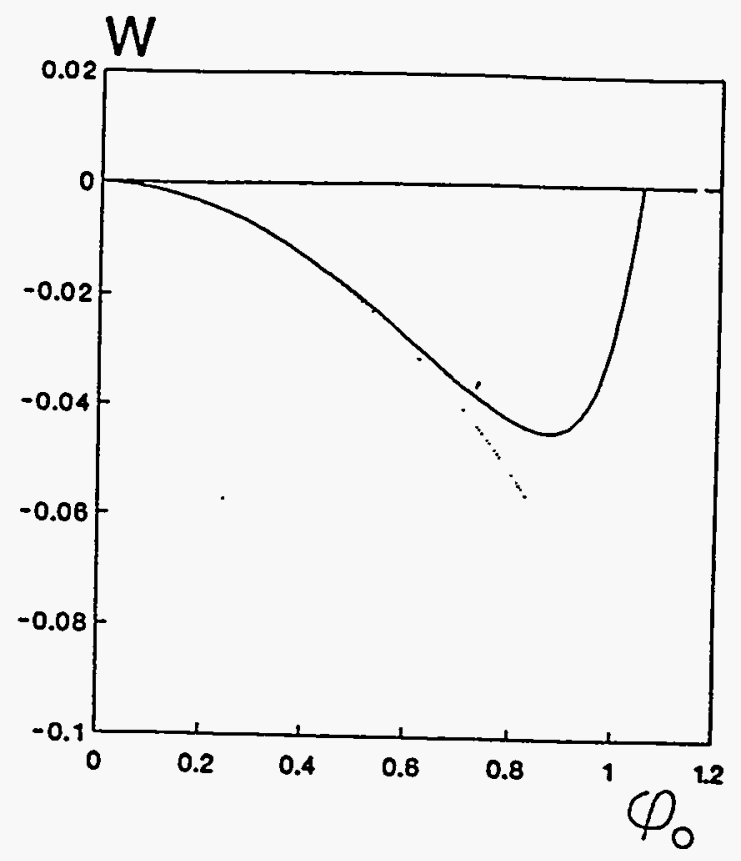

(d)

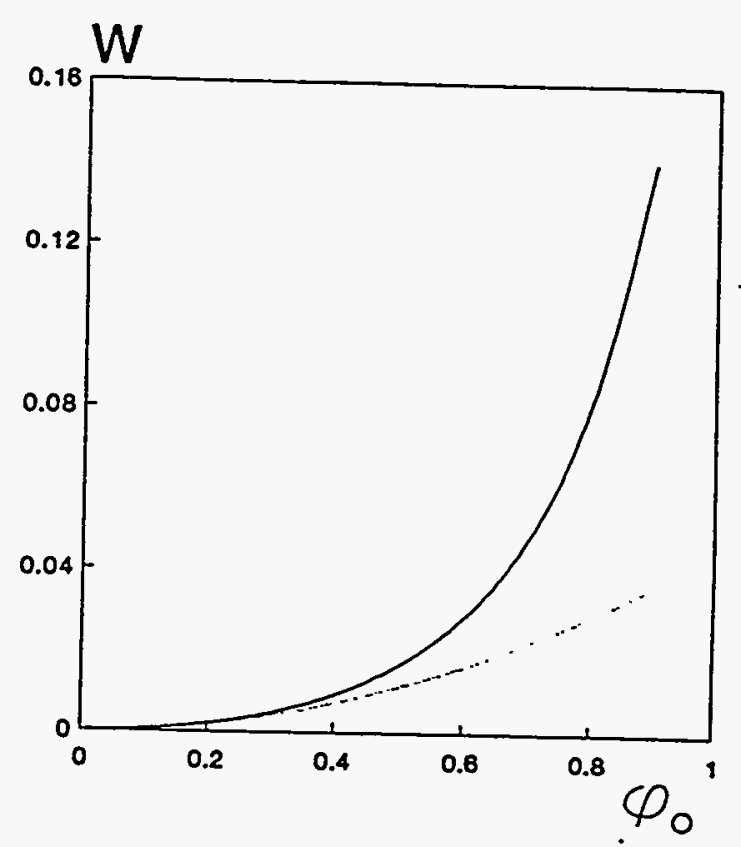

Figure 1.2: The energy variation $W / N$ plotted as a function of the amplitude $\varphi_{0}$ of the perturbed potential. The values of $W / N$ and $\varepsilon$ are measured in the units of $\varepsilon$ and $\varepsilon / e$, respectively. Curves relate to the following radii $R_{0}$ of plasma envelopes in the equilibrium state ( $R_{0}$ is normalized on the separatrix radius): $a-0.4 ; b-0.54 ; c$ $-0.6 ; d-0.8$. Parabolic profiles of the linear approximation are shown with dotted lines. 
gives way to the rise of $W$. However, for plasma envelopes with radii $R_{0}<0.54 R_{s}$, the energy $W$ remains negative for the arbitrary allowable amplitudes $\varphi_{0}$ (Fig. 1.2(a)). In the case, when $R_{0}=0.54 R_{s}$, the energy $W$ becomes zero for the maximum allowable potential amplitude (Fig. 1.2(b)). In the region $0.54 R_{s}<R_{0}<0.72 R_{s}$ the energy $W$ is found to be positive for the large amplitudes $\varphi_{0}$ (Fig. 1.2(c)), and hence the possibility of the "negative inertia" stabilization fails.

In the interval $0.72 R_{s}<R_{0}<R_{s}$, where according to the linear theory the energy $\mathrm{W}$ increases as $\varphi_{0}^{2}$, the nonlinear effects lead to even greater growth-rate of the profile $W\left(\varphi_{0}\right)$ (Fig. 1.2(d)). Thus in this case the energy $W$ occurs to be positive for all possible amplitudes. Consequently, the nonlinear stabilization due to the "negative inertia" effect in the interval of $R_{0}$ under examination turns to be impossible.

\subsection{Application to the beam-plasma neutron source}

Our results can be of some interest for the development of the beam-plasma neutron source (BPNS) [9]. In this system a relatively short mirror machine is filled with a cold tritium plasma which serves as a target for high energy ( $60 \mathrm{keV})$ deuterons which are produced by $\mathrm{NB}$ injection and confined in the same mirror machine. In order to reduce the heat losses through the cold plasma electrons, there is envisaged the use of the long solenoidal sections with gradually decreasing magnetic field between the mirror cell and the end-walls: as the target plasma is a collisional one and the heat flux is determined by the thermal conductivity, this arrangement indeed reduces the heat flux. Another implication of the using of these long sections is a considerable increase of the inertia of the flute perturbations. As one can easily show [13j, for the global mode the kinetic energy can be evaluated as

$$
\dot{\xi}^{2} \pi a_{0}^{2} B_{0}^{2} \int \frac{M n d l}{B^{2}},
$$

where $\xi$ is a plasma displacement in the equatorial plane of the device, $B_{0}$ and $a_{0}$ are magnetic field strength and plasma radius in this plane, respectively. Denoting the 
half-length of the mirror cell as $L$, one can obtain the following expression for the potential energy:

$$
\xi^{2}\left(n_{*} T_{*} / L^{2}\right) \pi a_{0}^{2} L,
$$

where the subscript " $*$ " refers to the high energy particles.

The growth-rate $\Gamma$ of the flute perturbations is equal to

$$
\frac{1}{L}\left(\frac{T_{*}}{M}\right)^{1 / 2}\left(\frac{n_{*} L}{B_{0}^{2} \int \frac{n d l}{B^{2}}}\right)^{1 / 2},
$$

while the drift frequency of the fast ions is

$$
\Omega_{d} \sim \frac{\rho_{*}}{L^{2}}\left(\frac{T_{*}}{M}\right)^{1 / 2}
$$

Here $\rho_{*}$ denotes the Larmor radius of the hot particle. After the substitution of the numerical values of all variables (see [9]), it turns out that

$$
\frac{\Gamma}{\Omega_{d}} \sim 0.1
$$

that is, the contribution of the fast ions to the potential energy of the flute perturbations in fact is zero. This should allow the change of the magnetic configuration from Yin-Yang to axisymmetric one, with the corresponding simplification of design of the neutron source.

\subsection{Conclusions}

In the present Chapter we have analyzed the stability of the axisymmetric mirror device with respect to purely electrostatic flute perturbations. We have investigated the scheme consisting of the long central cell conjugated with the end stabilizer that contains hot plasma. One of the main results of our research is the establishing of the 'fact, that a population of hot particles with fast azimuthal drift (see (1.4)) may pay a

- favorable contribution to the MHD stability of the system as the whole. The reason 
is that fast drifting particles affect not potential energy of MHD perturbations but rather their kinetic energy, and in the case, when the effective kinetic energy is found to be negative, the stabilization due to the "negative inertia" effect takes place.

Another system, to which our results can be applied, is a two-component plasma consisting of a cold dense background and a minority of hot particles that determines the plasma pressure (like in some versions of mirror-based neutron sources $[9,17]$ ). At high enough density of a cold component, condition (1.4) can easily be met.

We have also showed that the energy principle, formulated in [8:, can be generalized on the intermediate frequency interval $\Gamma / m \ll \Omega_{d} \leq \Gamma$ for the azimuthal modes with high numbers $m$ (see Appendix $C$ ). Therefore all conclusions, concerning the stabilization due to the "negative inertia" effect, can be transformed to these conditions as well.

\section{Appendices}

\section{A Van Dam-Rosenbluth-Lee energy principle in the case of the flute perturbations}

We start with the introducing the Clebsh coordinates $(\alpha, \theta)[15]$ with property

$$
\mathbf{B}=\nabla \alpha \times \nabla \theta
$$

The $\alpha$ coordinate is chosen so that the contour surfaces of constant $\alpha$ form a nested series of topological cylinders, and it is normalized to enclose the magnetic flux $2 \pi \alpha$ by any $\alpha$ surface. The $\theta$ coordinate is angle-like and of period $2 \pi$ on each $\alpha$ surface. In the limit of zero $\beta$ the magnetic field satisfies equation $\nabla \times \mathbf{B}=0$, and hence it can be expressed as a gradient of some potential $\chi$ :

$$
\mathbf{B}=\nabla \chi
$$


Vectors

$$
(\nabla \alpha, \nabla \theta, \nabla \chi)
$$

compose a covariant basis that we are going to deal with. We also define a contrvariant basis $(u, v, \tau)$, dual to $(1.58)$, in such a way that

$$
\begin{gathered}
\mathrm{u}=\frac{\nabla \theta \times \nabla \chi}{B^{2}}, \\
\mathrm{v}=-\frac{\nabla \alpha \times \nabla \chi}{B^{2}}, \\
\tau=\frac{\nabla \alpha \times \nabla \theta}{B^{2}} \equiv \frac{\mathrm{b}}{B} .
\end{gathered}
$$

According to the energy principle [7], derived within the assumption of the fast particle drift (see condition (1.4)), the energy variation $W$ consists of two terms:

$$
W=W_{f}+W_{k} .
$$

The first one, $W_{f}$, represents the local part of $W$ and can be written in the TaylorHastie form [14] :

$$
\begin{gathered}
W_{f}=\frac{1}{2} \int d^{3} x\left[\sigma Q_{\perp}^{2}+\zeta Q_{i \mathrm{i}}^{2}+\sigma j_{\|} \mathrm{b} \cdot(\xi \times \mathrm{Q})+q \xi \cdot \nabla^{\prime} p_{\|}\right. \\
\left.-(1 / B)\left(2 Q_{\|}+\xi \cdot \nabla B\right)\left(\xi \cdot \nabla^{\prime} p_{\perp}\right)\right]
\end{gathered}
$$

Here $\boldsymbol{\xi}$ is the displacement vector, $\mathbf{Q}$ is the Eulerian magnetic field perturbation,

$$
\mathrm{Q}=\boldsymbol{\nabla} \times(\boldsymbol{\xi} \times \mathrm{B})
$$

the subscripts $\|, \perp$ refer to the parallel and perpendicular components with respect to the direction of the unperturbed magnetic field, and the coefficients $\sigma$ and $\zeta$,

$$
\sigma=1-B^{-1}\left(\partial p_{\|} / \partial B\right), \quad \zeta=1-\left(\partial^{2} p_{\|} / \partial B^{2}\right)
$$

are measures of stability against firehose and mirror anisotropy modes, respectively.

- Also, the following notations are introduced:

$$
\nabla^{\prime}=\nabla-(\nabla B) \partial / \partial B
$$




$$
q=\mathrm{bb}: \boldsymbol{\nabla} \boldsymbol{\xi}
$$

The kinetic contribution to the energy variation, $W_{k}$, originated from the fast drifting particles, is given by

$$
W_{k}=-\frac{1}{2} \int d \alpha d \theta d \mu d J\left[\left(\frac{\partial F}{\partial \varepsilon}\right)_{J}\langle\langle H\rangle\rangle^{2}+\left(\frac{\partial J}{\partial \varepsilon}\right)\left(\frac{\partial F}{\partial J}\right)_{\varepsilon}\langle H\rangle^{2}\right],
$$

where

$$
H=-m v_{\|}^{2} q-\mu B(\nabla \cdot \xi-q),
$$

and $F=F(J(\alpha, \theta, \varepsilon, \mu), \varepsilon, \mu)$ is the equilibrium distribution function, depending only on the integrals of motion (see e.g. [16]). Single angle brackets in (1.62) describe the bounce average:

$$
\langle\ldots\rangle=\left(\frac{\partial J}{\partial \varepsilon}\right)^{-1} \oint d l v_{\|}^{-1}(. .)
$$

while double angle brackets denote the average both over bounce and drift motions:

$$
\langle\langle\ldots\rangle\rangle=\left(\frac{\partial \tilde{\Phi}}{\partial \varepsilon}\right)_{J}^{-1} \oint d \theta\left(\frac{\partial \varepsilon}{\partial \alpha}\right)_{J}^{-1}\langle\ldots\rangle
$$

where $\bar{\Phi}=\oint d \theta \alpha(\theta)$ is the flux adiabatic invariant $(\alpha=\alpha(\theta)$ defines the particle drift-surface, and integration is performing with $J$ and $\varepsilon$ being constant), $(\partial \tilde{\Phi} / \partial \varepsilon)_{J}$ is the precessional drift period, and $(\partial \varepsilon / \partial \alpha)_{J}=\langle d \beta / d t\rangle$ is the bounce-averaged rate of precession [10].

Now we turn to the calculation of the energy (1.59) for the flute-like perturbations, characterized by the following displacement vector $\xi$ :

$$
\begin{gathered}
\xi=\mathrm{b} \xi_{\|}+\xi_{\perp}, \\
\xi_{\perp}=\frac{\mathrm{B} \times \nabla \eta}{B^{2}}
\end{gathered}
$$

with function $\eta$ constant along the field line,

$$
\eta=\eta(\alpha, \theta)
$$

Since

$$
\nabla \eta=\frac{\partial \eta}{\partial \alpha} \nabla \alpha+\frac{\partial \eta}{\partial \theta} \nabla \theta
$$


the perpendicular displacement (1.66) can be expressed as

$$
\boldsymbol{\xi}_{\perp}=-\frac{\partial \eta}{\partial \theta} \mathbf{u}+\frac{\partial \eta}{\partial \alpha} \mathbf{v}
$$

In the curved coordinate system $(\alpha, \theta, l)$ (with $l$ as a coordinate along the field line, determined by $d l=d \chi / B$ ) one obtains:

$$
\begin{aligned}
\nabla \cdot \xi & =B \frac{\partial}{\partial l}\left(\frac{\xi_{\|}}{B}\right)+B^{2}\left[\frac{\partial}{\partial \theta}\left(\frac{1}{B^{2}} \frac{\partial \eta}{\partial \alpha}\right)-\frac{\partial}{\partial \alpha}\left(\frac{1}{B^{2}} \frac{\partial \eta}{\partial \theta}\right)\right]= \\
& =B \frac{\partial}{\partial l}\left(\frac{\xi_{\|}}{B}\right)-\frac{2}{B}\left[\frac{\partial B}{\partial \theta} \frac{\partial \eta}{\partial \alpha}-\frac{\partial B}{\partial \alpha} \frac{\partial \eta}{\partial \theta}\right] .
\end{aligned}
$$

As it follows from (1.61), (1.65), the expression for $q$ yields:

$$
q=\frac{\partial \xi_{\|}}{\partial l}-\boldsymbol{\xi}_{\perp} \cdot(\mathbf{b} \cdot \nabla) \mathbf{b}
$$

Accounting for $\nabla \times B=0$, and using the relationships

$$
\begin{gathered}
(\mathbf{b} \cdot \nabla) \mathbf{b}=-\mathbf{b} \times(\nabla \times \mathbf{b}), \\
\nabla \times \mathbf{b}=-\frac{1}{B}(\nabla B \times \mathbf{b}),
\end{gathered}
$$

we come to

$$
q=\frac{\partial \xi_{\|}}{\partial l}-\frac{1}{B}\left[\frac{\partial B}{\partial \theta} \frac{\partial \eta}{\partial \alpha}-\frac{\partial B}{\partial \alpha} \frac{\partial \eta}{\partial \theta}\right]
$$

After simple manipulations one can find from (1.63), (1.68), (1.69) that

$$
\begin{gathered}
\langle H\rangle=\left(\frac{\partial J}{\partial \varepsilon}\right)^{-1} \int \frac{d l}{v_{\|}} \xi_{\|} \frac{\partial}{\partial l}\left(\frac{M v_{\|}^{2}}{2}+\mu B\right)+ \\
+\left(\frac{\partial J}{\partial \varepsilon}\right)^{-1} \int \frac{d l}{B v_{: !}}\left(M v_{\|}^{2}+\mu B\right)\left[\frac{\partial B}{\partial \theta} \frac{\partial \eta}{\partial \alpha}-\frac{\partial B}{\partial \alpha} \frac{\partial \eta}{\partial \theta}\right] .
\end{gathered}
$$

The contribution from the first integral vanishes, since the particle energy conserves along the field line.

As it was shown in [15],

$$
\frac{\partial J}{\partial \alpha}=-\int \frac{d l}{B v_{\|}}\left(M v_{\|}^{2}+\mu B\right) \frac{\partial B}{\partial \alpha}
$$




$$
\frac{\partial J}{\partial \theta}=-\int \frac{d l}{B v_{\|}}\left(M v_{\|}^{2}+\mu B\right) \frac{\partial B}{\partial \theta} .
$$

Combining (1.71),(1.72) with (1.70), one can rewrite the expression for $\langle H\rangle$ as

$$
\langle H\rangle=-\left(\frac{\partial J}{\partial \varepsilon}\right)^{-1} \frac{\partial J}{\partial \theta} \frac{\partial \eta}{\partial \alpha}+\left(\frac{\partial J}{\partial \varepsilon}\right)^{-1} \frac{\partial J}{\partial \alpha} \frac{\partial \eta}{\partial \theta}
$$

Now it is easy to perform the average of $\langle H\rangle$ over the drift motion. Inserting (1.73) into (1.64) and taking into account that

$$
\left(\frac{\partial \alpha}{\partial \theta}\right)_{J}=-\frac{\partial J}{\partial \theta}\left(\frac{\partial J}{\partial \alpha}\right)^{-1},\left(\frac{\partial \varepsilon}{\partial \alpha}\right)_{J}=-\frac{\partial J}{\partial \alpha}\left(\frac{\partial J}{\partial \varepsilon}\right)^{-1}
$$

we have:

$$
\langle\langle H\rangle\rangle=\left(\frac{\partial \bar{\Phi}}{\partial \varepsilon}\right)_{J}^{-1} \oint\left(d \theta \frac{\partial \eta}{\partial \theta}+d \alpha \frac{\partial \eta}{\partial \alpha}\right)=\left(\frac{\partial \Phi}{\partial \varepsilon}\right)_{J}^{-1} \oint d \eta=0 .
$$

Finally, using

$$
\left(\frac{\partial F}{\partial \alpha}\right)_{e}=-\left(\frac{\partial J}{\partial \varepsilon}\right)\left(\frac{\partial \varepsilon}{\partial \alpha}\right)_{J}\left(\frac{\partial F}{\partial J}\right)_{\varepsilon}
$$

one obtains after the substitution of (1.73) into (1.62):

$$
\begin{gathered}
W_{k}=-\frac{1}{2} \int d \alpha d \theta d \mu d \varepsilon\left(\frac{\partial J}{\partial \alpha}\right)\left(\frac{\partial F}{\partial \alpha}\right)_{\epsilon}\left(\left(\frac{\partial \eta}{\partial \theta}\right)^{2}-\right. \\
\left.-2\left(\frac{\partial J}{\partial \theta}\right)\left(\frac{\partial J}{\partial \alpha}\right)^{-1}\left(\frac{\partial \eta}{\partial \alpha}\right)\left(\frac{\partial \eta}{\partial \theta}\right)+\left(\frac{\partial J}{\partial \alpha}\right)^{-2}\left(\frac{\partial J}{\partial \theta}\right)^{2}\left(\frac{\partial \eta}{\partial \alpha}\right)^{2}\right) .
\end{gathered}
$$

Here we have changed the set of the integration variables, and perform the integration over $d \varepsilon$ instead of $d J$.

Now we turn to the calculation of the energy variation $W_{f}$. Since $W_{f}$ does not depend on the parallel component $\xi_{\|}$(see [14]), and besides that the displacement (1.65) does not perturb the magnetic field, $Q=0$, the expression (1.60) reduces to

$$
\begin{gathered}
W_{f}=-\frac{1}{2} \int d^{3} x\left(\frac{1}{B}\left(\xi_{\perp} \cdot \nabla B\right)\left(\xi_{\perp} \cdot \nabla^{\prime} p_{\perp}\right)+\right. \\
\left.+\frac{1}{B}\left(\xi_{\perp} \cdot \nabla^{\prime} p_{\|}\right)\left[\frac{\partial B}{\partial \theta} \frac{\partial \eta}{\partial \alpha}-\frac{\partial B}{\partial \alpha} \frac{\partial \eta}{\partial \theta}\right]\right) .
\end{gathered}
$$


The parallel and perpendicular pressure components, presented in (1.75), are given by

$$
\begin{aligned}
& p_{\|}=\int \frac{d \varepsilon d \mu}{v_{\|}} B M v_{\mathrm{li}}^{2} F, \\
& p_{\perp}=\int \frac{d \varepsilon d \mu}{v_{\|}} B \mu B F .
\end{aligned}
$$

The substitution of (1.67) into (1.75) leads to

$$
\begin{aligned}
W_{f}= & \frac{1}{2} \int \frac{d^{3} x}{B}\left\{\frac{\partial B}{\partial \alpha}\left[\frac{\partial P}{\partial B} \frac{\partial B}{\partial \alpha}-\frac{\partial P}{\partial \alpha}\right]\left(\frac{\partial \eta}{\partial \theta}\right)^{2}+\frac{\partial B}{\partial \theta}\left[\frac{\partial P}{\partial B} \frac{\partial B}{\partial \theta}-\frac{\partial P}{\partial \theta}\right]\left(\frac{\partial \eta}{\partial \alpha}\right)^{2}-\right. \\
& \left.-\frac{\partial B}{\partial \alpha}\left[\frac{\partial P}{\partial B} \frac{\partial B}{\partial \theta}-\frac{\partial P}{\partial \theta}\right] \frac{\partial \eta}{\partial \theta} \frac{\partial \eta}{\partial \alpha}-\frac{\partial B}{\partial \theta}\left[\frac{\partial P}{\partial B} \frac{\partial B}{\partial \alpha}-\frac{\partial P}{\partial \alpha}\right] \frac{\partial \eta}{\partial \theta} \frac{\partial \eta}{\partial \alpha}\right\}
\end{aligned}
$$

where $P=p_{\|}+p_{\perp}$,

$$
P=\int \frac{d \varepsilon d \mu B}{v_{\|}}\left(M v_{\|}^{2}+\mu B\right) F(\mu, \varepsilon, J) .
$$

The calculation of the derivatives of $P$, entering into (1.76), gives:

$$
\begin{gathered}
\frac{\partial P}{\partial B}=\int d \varepsilon d \mu F \frac{\partial}{\partial B}\left(B \frac{\left(M v_{\|}^{2}+\mu B\right)}{v_{\|}}\right), \\
\frac{\partial P}{\partial \alpha}=\frac{\partial B}{\partial \alpha} \int d \varepsilon d \mu F \frac{\partial}{\partial B}\left(B \frac{\left(M v_{\|}^{2}+\mu B\right)}{v_{\|}}\right)+\int \frac{d \varepsilon d \mu B}{v_{\|}}\left(M v_{\|}^{2}+\mu B\right)\left(\frac{\partial F}{\partial \alpha}\right)_{e},(1.7) \\
\frac{\partial P}{\partial \theta}=\frac{\partial B}{\partial \theta} \int d \varepsilon d \mu F \frac{\partial}{\partial B}\left(B \frac{\left(M v_{\|}^{2}+\mu B\right)}{v_{\|}}\right)+\int \frac{d \varepsilon d \mu B}{v_{\|}}\left(M v_{\|}^{2}+\mu B\right)\left(\frac{\partial F}{\partial \theta}\right)_{c} .
\end{gathered}
$$

Now reminding that $d^{3} x=d \alpha d \theta d l / B$, and accounting for (1.71), (1.72), (1.77), one can transform (1.76) to

$$
\begin{gathered}
W_{f}=\frac{1}{2} \int d \varepsilon d \mu d \alpha d \theta\left\{\frac{\partial J}{\partial \alpha}\left(\frac{\partial F}{\partial \alpha}\right)_{e}\left(\frac{\partial \eta}{\partial \theta}\right)^{2}+\right. \\
\left.+\frac{\partial J}{\partial \theta}\left(\frac{\partial F}{\partial \theta}\right)_{e}\left(\frac{\partial \eta}{\partial \alpha}\right)^{2}-\left(\frac{\partial J}{\partial \theta}\left(\frac{\partial F}{\partial \alpha}\right)_{e}+\frac{\partial J}{\partial \alpha}\left(\frac{\partial F}{\partial \theta}\right)_{e}\right) \frac{\partial \eta}{\partial \theta} \frac{\partial \eta}{\partial \alpha}\right\}
\end{gathered}
$$

The latter expression, together with equations

$$
\left(\frac{\partial F}{\partial \theta}\right)_{e}=\frac{\partial J}{\partial \theta}\left(\frac{\partial F}{\partial J}\right)_{e},\left(\frac{\partial F}{\partial \alpha}\right)_{e}=\frac{\partial J}{\partial \alpha}\left(\frac{\partial F}{\partial J}\right)_{e},
$$




$$
\left(\frac{\partial F}{\partial \theta}\right)_{\varepsilon}=\frac{\partial J}{\partial \theta}\left(\frac{\partial J}{\partial \alpha}\right)^{-1}\left(\frac{\partial F}{\partial \alpha}\right)_{\varepsilon},
$$

allows one to show easily that $W_{f}$ equals to the expression (1.74) with the inverse sign, and hence the energy variation (1.59) is found to be identically zero.

\section{B Energy principle for the "disk"-like plasma}

Consider the limit of zero longitudinal hot plasma pressure, $p_{\|}=0$, when transverse energy of hot particles greatly exceeds their longitudinal energy. In the equilibrium state these particles perform small bounce oscillations round minimum field point, so that

$$
\varepsilon-\mu B_{0} \ll \mu B_{0},
$$

$B_{0}$ marks minimum value of the field strength on the given field line. Using expansion of $B$ along the field line in the vicinity of $B_{0}$,

$$
B \simeq B_{0}+\tilde{B} l^{2},
$$

one can carry out the integration in (1.10) explicitly:

$$
J=\pi \sqrt{2 M} \frac{\Delta \varepsilon}{\sqrt{\mu \tilde{B}}},
$$

with $\Delta \varepsilon=\varepsilon-\mu B_{0}$. Now it would not be difficult to calculate with sufficient accuracy $\varepsilon$ - and $\Phi$ - derivatives of $J$ :

$$
\begin{gathered}
J_{\varepsilon}=\frac{J}{\Delta \varepsilon}, \quad J_{\Phi}=-\mu B_{0 \Phi} \frac{J}{\Delta \varepsilon}, \\
J_{\varepsilon \varepsilon}=0, \quad J_{c \Phi}=-\frac{\tilde{B}_{\Phi}}{\tilde{B}} \frac{J}{\Delta \varepsilon}, \\
J_{\Phi \Phi}=-\mu B_{0 \Phi \Phi} \frac{J}{\Delta \varepsilon}+\mu B_{0 \Phi} \frac{\bar{B}_{\Phi}}{\tilde{B}} \frac{J}{\Delta \varepsilon} .
\end{gathered}
$$

Here we have taken into account, that $\Delta \varepsilon / \mu B_{0}$ is a small parameter according to (1.79). Substitution of (1.81) into (1.24) leads to the following expression for the 
energy variation:

$$
W=\frac{e^{2}}{4} \int d \mu d \Phi \frac{F}{\mu} \frac{d}{d \Phi}\left(\frac{\tilde{\varphi}^{2}}{B_{0 \Phi}}\right) .
$$

Note that there is no integration over $d \varepsilon$ in (1.82). The reason is that in the case under consideration, the distribution function $F$ depends on $\varepsilon$ approximately in a $\delta$ functional way, $F \sim \delta\left(\varepsilon-\mu B_{0}\right)$, and so the integration over $d \varepsilon$ can be done explicitly.

\section{Generalization of the energy principle for the case: $\Gamma / m \ll \Omega_{d} \leq \Gamma$}

The crucial point of the derivation of the expression for the energy variation in paper [8] (as well as in [7]) is the exploitation of the fact that the flux adiabatic invariant conserves. As is well known (see, e.g. [10]), when the frequency of the drift motion $\Omega_{d}$ around the axis is high as compared with the inverse characteristic time of variation of the electric and magnetic fields, then the magnetic flux $\bar{\Phi}$ encompassed by the drift surface is an adiabatic invariant (notation $\bar{\Phi}$ shouldn't be confused with a flux coordinate $\Phi$, an independent variable). In the case considered in the present paper, the magnetic field is constant; the varying is electrostatic potential $\varphi$ of the perturbations. The slowness of variation of $\varphi$ in the above mentioned sense (see (1.4)) guarantees the conservation of $\bar{\Phi}$.

When considering the case of perturbations with high azimuthal mode number $m \gg 1$, one may encounter the situation when the drift frequency $\Omega_{d}$ is lower then $\Gamma$ but higher than $\Gamma / m$ :

$$
\Gamma / m \ll \Omega_{d} \leq \Gamma
$$

The inequality $\Omega_{d} \leq \Gamma$ means that one cannot thoughtlessly use the traditional adiabatic invariant $\tilde{\Phi}$. However, we shall show that, under the condition $\Gamma / m \ll \Omega_{d}$, there exists another adiabatic invariant, similar to $\bar{\Phi}$.

We consider the potential perturbation (not necessarily small) which is of the form (1.8) and changes in time with the characteristic frequency $\Gamma$. Then the inequality 
$\Gamma / m \ll \Omega_{d}$ means that the guiding centre traverses one spatial period of the system in a time that is short as compared to the time of potential variation. Just this fact is a basis for existence of the generalized adiabatic invariant.

The guiding centre motion, averaged over the fast bouncing along the field line, is governed by the following equations [11]:

$$
\begin{gathered}
\dot{\psi}=-\frac{2 \pi c}{e t_{\|}} J_{\Phi}, \\
\dot{\Phi}=\frac{2 \pi c}{e t_{\|}} J_{\psi},
\end{gathered}
$$

where $t_{\|}=J_{c}$ is the transit time between the turning points. In the context of the problem under consideration, $J$ is a constant of motion (as well as $\mu$ ). Resolving the equation $J=$ const with respect to $\varepsilon$, we can find the function $\varepsilon(\psi, \Phi)$ for a given particle. By differentiating the function $J(\varepsilon(\psi, \Phi), \Phi, \psi)$ over $\psi$ and $\Phi$, we find:

$$
\frac{\partial \varepsilon}{\partial \psi}=-\frac{J_{\psi}}{J_{e}}, \quad \frac{\partial \varepsilon}{\partial \Phi}=-\frac{J_{\Phi}}{J_{e}},
$$

and the equations of motion (1.84) acquire the Hamiltonian form:

$$
\begin{gathered}
\dot{\psi}=\frac{2 \pi c}{\epsilon} \frac{\partial \varepsilon}{\partial \Phi}, \\
\dot{\Phi}=-\frac{2 \pi c}{e} \frac{\partial \varepsilon}{\partial \psi},
\end{gathered}
$$

with $\psi$ and $\Phi$ being canonically conjugate variables and $\varepsilon$ playing a role of Hamiltonian. As the explicit dependence of $\varepsilon$ on $t$ is slow in the sense that the guiding centre traverses one spatial period of the system (in coordinate $\psi$ ) in a time short as compared to the time of potential variation, equations (1.85) possess an adiabatic invariant

$$
\tilde{\Phi}^{\star}=\int_{T_{\psi}} \Phi d \psi,
$$

where integration is carried out over one spatial period $T_{\psi}$, equal to $2 \pi / m$. Of course, for slow enough potential variations, $\Gamma \ll \Omega_{d}$, the integration in (1.86) can be extended to a full rotation of a particle around the axis, and (1.86) reduces to the standard flux invariant. 
Now it becomes clear that the expression for the energy variation, that takes into account conditions (1.83), coincides with formula (1.24). Indeed, all calculations in this case would exactly repeat calculations, performed in Section 1.2, with the only exclusion: $\bar{\Phi}$ conservation requirement should be replaced by the requirement of the conservation of $\tilde{\Phi}^{\star}$. But this changing doesn't, obviously, affect formula (1.24), since the averaging of the expressions, composed of function (1.8), either over $2 \pi / m$-interval (as in (1.86)) or over $2 \pi$-interval (as in the expression for $\bar{\Phi}$ ) would finally lead to the same result. 


\section{Bibliography}

[1] D.D. Ryutov, G.V. Stupakov, JETP Lett., 42, 29 (1985).

[2] D.D. Ryutov, G.V. Stupakov, Sov.J. Plasma Phys., 12, 815 (1986).

[3] G.I. Dimov, Proceedings of 10th European School on Plasma Physics, Tbilisi, 157 (1991).

[4] M.N. Rosenbluth, N.A.Krall, N.Rostoker, Nucl. Fusion, Suppl. Pt. 1, 143 (1962).

[5: M.D. Kruskal, C.R. Oberman, Phys. Fluids, 1, 275 (1958).

[6! V.P. Nagormyj, D.D. Ryutov, G.V. Stupakov, Preprint N-83-74, Institute of Nuclear Physics, Novosibirsk (1983).

[7] J.W. Van Dam, M.N. Rosenbluth, Y.C. Lee, Phys. Fluids, 25, 1349 (1982).

[8] I.M. Lansky, D.D. Ryutov, JETP Letters, 57, No.1, 30 (1992).

[9] F.H. Coensgen, T.A. Casper, D.L. Correl, et al., Rep. UCRL-97280, Rev. 1, Lawrence Livermore National Laboratory (1987).

[10] T.G. Northrop, E. Teller, Phys. Rev., 117, 215 (1960).

[11] A.I. Morozov, L.S. Solov'ev, Reviews of Plasma Physics, 2, Consultants Burcau, New York (1966).

[12] D.D. Ryutov, G.V. Stupakov, Reviews of Plasma Physics, 13, Consultants Bureau, New York - London, 93 (1987). 
[13] V.P. Nagornyj, D.D. Ryutov, G.V. Stupakov, Nucl. Fusion, 24, No. 11, 1421 (1984).

[14] J.B. Taylor, R.J. Hastie, Phys.Fluids, 8, 232 (1965).

[15] W.A. Newcomb, J. Plasma Physics, 26, part 3, 529 (1981).

[16] R.J. Hastie, J.B. Taylor, F.A. Haas, Ann.Phys. (NY), 41, 302 (1967).

[17] V.V. Mirnov, V.P. Nagormyj, D.D. Ryutov, Preprint N-81-40. Institute of Nuclear Physics, Novosibirsk (1984). 


\section{Chapter 2}

\section{Non-paraxial axisymmetric MHD}

\section{anchors}

Big attention is paid last years to search for axisymmetric configurations of magnetic field which can provide MHD stable plasma confinement [1,7]. By obvious reasons, preference is given to the configurations with "natural" singlyconnected region of plasma confinement. Perspective for this direction of research has been demonstrated in [i] where non-paraxial mirror trap, formed by a system of two small coils (i.e., dipoles) in an external relatively weak homogeneous magnetic field, has been pointed out to be the example of stable axisymmetric configuration. Inserting of non-paraxial mirror trap into the composition of an open (for instance, an ambipolar) device guaranties stability of large-scale flute perturbations, while small-scale perturbations are supposed to be stabilized due to Finite Larmor Radius effects. In this chapter, we poit out a method of search for axisymmetric configurations which provide stability of rigid ( $m=1$ ) flute mode and prove several theorems, elucidating the effect of non-paraxiality on sloshing ions stabilization of flute modes $[3,4$. 


\subsection{Stability of disk-shaped plasma}

Let us, first, consider disk-shaped plasma with $p_{\|}=0$, confined at the equatorial plane $z=0$ of non-paraxial mirror trap. For the sake of simplicity, we assume for the trap to be symmetrical about the plane $z=0$. Such system is stable against global flute mode provided that there exist at least one point along radius, near which the following two inequality are simultaneously satisfied [7]:

$$
\begin{gathered}
\frac{B_{0}}{r} \frac{d}{d r} r \frac{d B_{0}}{d r} \leq 2\left(\frac{d B_{0}}{d r}\right)^{2}, \\
\frac{d}{d r} \frac{\Phi}{r B_{0}} \frac{d B_{0}}{d r} \geq 0
\end{gathered}
$$

where $B_{0}(r)=B_{z}(r, 0)$ is the magnetic field at the equatorial plane,

$$
\Phi=2 \pi \int_{0}^{r} d r r B_{0}(r)
$$

is the magnetic flux throughout the circle of radius $r$. The first inequality (2.1) guaranties the existence of "longitudinal" minimum of magnetic field near $z=0$ plane and, hence, stability against plasma displacement along field lines. The second inequality provides stability against global displacements of the plasma across magnetic field. In the region, close to plasma axis as well as very distant from it, the conditions (2.1), (2.2) are incompatible [ 7$]$. Therefore the region, selected by these inequalities, if it exists, has form of a ring. The above mentioned system of two magnetic dipoles with superimposed weak homogeneous external magnetic field gives first example of such "ring of stability". Trying to ascertain which magnitude of the external field corresponds to arising the ring of stability readily gives a common guide of search for the system with the ring of stability.

\subsubsection{System of two magnetic dipoles}

Magnetic dipole $\mathrm{m}$ produces magnetic field

$$
\mathrm{B}=\frac{3(\mathrm{mR}) \mathrm{R}-R^{2} \mathrm{~m}}{R^{5}}
$$


at the point with radius-vector $\mathrm{R}$. First of all, we note that own magnetic field of pair of similar dipoles, directed along the axis $z$ wich connects them, goes to zero at the equatorial plane $z=0$ on a distance from the axis. If to choose a half of distance between the dipoles for the unit of length, then from (2.3) we get

$$
B_{0}(r)=B_{*} \frac{2-r^{2}}{2\left(1+r^{2}\right)^{5 / 2}}
$$

where $B_{*}=4 m$ is the field at the center $r=z=0$ of coordinates, so that the null of the magnetic field is the circle of the radius $r=\sqrt{2}$. A separatrice surface matches the null and separates the regions with reverse direction of magnetic field B. If to superimpose weak homogeneous magnetic field $\mathrm{H}$, co-directed with the field $\mathrm{B}$ on the axis $z$ of the dipoles system, the circle of second null, initially (at $\mathrm{H}=0$ ) placed at infinity $(r=\infty)$ in the equatorial plane, approaches to the first null and merges it near the radius $r=2$ when $H / B_{0}=5^{-5 / 2} \simeq 1.79$. Simultaneously with the merging, the regions of reversely directed magnetic field disappear (see Fig. 2.1). lot specifying further our chose of magnetic system, we show that it is the moment of merging when the ring of stability appears for disk-shaped plasma.

Iie can neglect the effects connected with cylindrical geometry of the problem and consider the magnetic field to be planar near null rings. Collating cylindrical coordinates $\{r, \varphi, z\}$ to Cartesian ones $\{x, y, z\}$, we can write the expansion of scalar magnetic potential $\psi$, which satisfies to the equation $\Delta \psi=0$, in the following form:

$$
\psi=H z-\alpha x z+\beta\left(x^{2} z-\frac{1}{3} z^{3}\right)+\gamma\left(x^{2}-z^{2}\right)+\nu\left(x z^{2}-\frac{1}{3} x^{3}\right) .
$$

With ascertaining the symmetry about the plane $z=0$, the coefficients $\gamma, \nu$ should be equalized to zero. The coefficient $\alpha$ can be converted into zero by means of changing the center of coordinates by $\alpha / 2, \beta$ along the axis $x$ and redefining $H$ to $H+\alpha^{2} / 4 \beta$. After this, the magnetic field $\mathrm{B}=\nabla \psi$ remains depending on the two parameters $H$ and $\beta$ only:

$$
B_{x}=2 \beta x z, \quad B_{y}=0, \quad B_{z}=H+\beta x^{2}-\beta z^{2},
$$



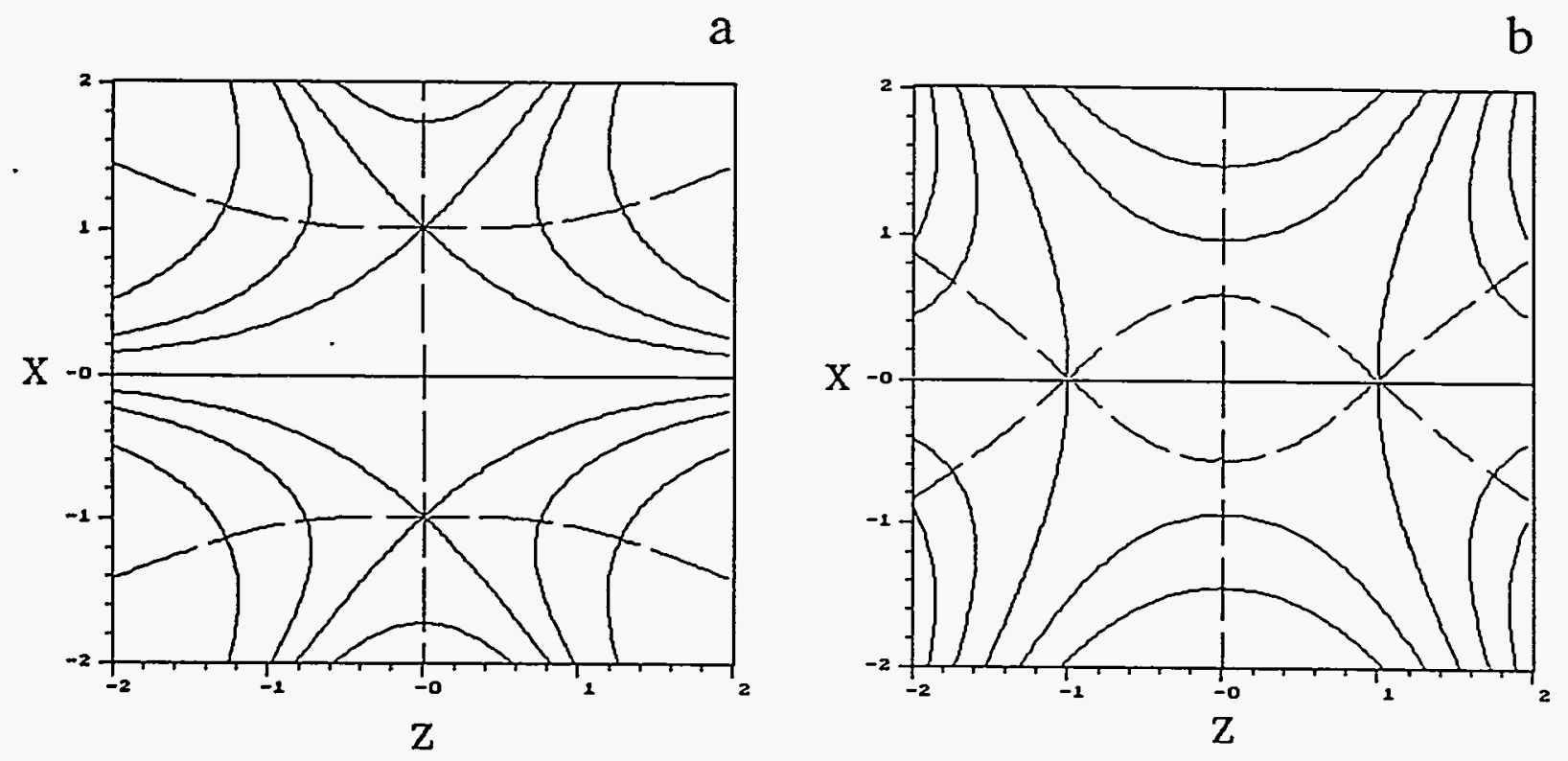

Figure 2.1: Map of magnetic field near null. $a-H / \beta=-1, b-H / \beta=+1$. 
with one of them, for instance $\beta$, being positive. With the approach (2.6) used, field lines are cubic hyperbolas. They are described by the following equation

$$
\phi=\phi_{0}+H x+\beta\left(\frac{1}{3} x^{3}-x z^{2}\right)=\text { const }
$$

and are shown on Fig. 2.1. The magnetic field in the equatorial plane

$$
B_{0}(x)=H+\beta x^{2}
$$

has the extremum, equal to $H$. If $H / \beta<0$, magnetic field nulls lay in the equatorial plane (see Fig. 2.1,a):

$$
x_{1,2}= \pm \sqrt{-\frac{H}{\beta}}, \quad z_{1,2}=0,
$$

while they are plased symmetrically on both sides from it if $H / \beta>0$ (see Fig. 2.1,b):

$$
x_{1,2}=0, \quad z_{1,2}= \pm \sqrt{\frac{H}{\beta}} .
$$

The inequalities (2.1), (2.2), which determine the boundaries of stability ring, are transformed in the case of planar field by means of limiting transition to the form

$$
\left(\frac{d B_{0}}{d x}\right)^{2} \leq B_{0} \frac{d^{2} B_{0}}{d x^{2}} \leq 2\left(\frac{d B_{0}}{d x}\right)^{2} .
$$

Inserting here $B_{0}$ from (2.8), we get the condition

$$
\frac{1}{3} \frac{H}{\beta} \leq \beta x^{2} \leq \frac{H}{\beta},
$$

which can be satisfied when $H / \beta>0$ only: For $H / \beta>0$ there are two solutions; however one of them corresponds to outer ring of stability; which is separated from plasma's axis by the separatrix. Outer boundaries $x= \pm \sqrt{H / \beta}$ of the couple of rings are fixed by the condition of stability of global displacement, while the internal boundaries $x= \pm \sqrt{H / 3 \beta}$ by the condition of stability of longitudinal displacements. The configuration of the kind, shown on Fig. 2.1,b, can be created by two methods. In the first variant, the coils marked by the plus sign on Fig. $2.1, \mathrm{~b}$, produce on their axis magnetic field co-directed with the external quasi-homogeneous field. This is 
configuration of non-paraxial mirror kind. In the alternative variant, the coils, marked by the minus sign, produce anti-directed magnetic field, forming the configuration of divertor kind. Dashed line on Fig. 2.1,b connects the extremums magnetic field along field lines, i.e., the points where $(B, \nabla) B=0$. The magnetic field achieves its maximum on the piece $A B$ and its minimum on every other pieces, including the line $O A O^{\prime}$. Hence, longitudinal stability is provided on the piece $O A O^{\prime} ;$ however, the condition of global displacement stability is not fulfilled there. Thus, the ring of stability exists only near the equatorial plane. In the divertor variant, the ring of stability can get close to the plasma axis, which lays below bottom edge of thr Fig. 2.1; when this occurs, the ring of stability disappears and two separate mirrors appear instead of the divertor.

As it was already pointed out, merging of nulls and, hence, formation of stability rings in the system of two dipoles occurs for very weak external field. However, from practical point of view, the opposite case, where non-paraxial anchor field is less than external field of solenoidal part of an open trap, is more interesting. Considering for the ratio $b$ of anchor's magnetic field to external one to be small, we can rewrite the inequalities (2.1), (2.2) in the form

$$
\left(\frac{d b}{d r}\right)^{2}-\frac{1}{r^{3}}\left(\frac{d b}{d r}-r \frac{d^{2} b}{d r^{2}}\right) \int_{0}^{r} d r r^{2} \frac{d b}{d r} \leq \frac{d^{2} b}{d r^{2}}+\frac{1}{r} \frac{d b}{d r} \leq 2\left(\frac{d b}{d r}\right)^{2} .
$$

As $b \rightarrow 0$, the inequalities (2.13) can be satisfied in narrow interval close to the radius $r_{0}$, such that

$$
r d^{2} b / d r^{2}+d b / d r=0 .
$$

Having substituted $r d^{2} b / d r^{2}$ in the left-hand-side of inequalities chain (2.13) by $-d b / d r$, we easy ascertain that it is less than the right-hand-side if

$$
\left(\frac{d b}{d r}\right)^{2}+\frac{2}{r^{3}} \frac{d b}{d r} \int_{0}^{r} d r r^{2} \frac{d b}{d r} \geq 0 .
$$

Among all solutions $r_{0}$ of the equation (2.14), the inequality (2.15) separates those, which correspond to ring of stability. Using (2.4) we find that, in the case of dipoles 
system, the equation (2.14) has two solutions $r_{0}=2(1 \pm 1 / \sqrt{3})^{1 / 2}$, but only one of them, namely $r_{0}=2(1-1 / \sqrt{3})^{1 / 2} \simeq 0.85$, satisfies to the inequality (2.15). This solution does not depend on sign of $b$, i.e., corresponding ring of stability exists at any direction of external field about the dipoles' magnetic filed.

Let us show that the ring of stability is separated by separatrix from the plasma's axis $r=0$ if $B_{*} / H>0$, i.e., if external magnetic field is opposite to the direction of dipoles' filed at their axis. The separatrix is the filed line which goes through the nulls. When $B_{*} / H \rightarrow+0$, magnetic filed nulls are placed near the dipoles. Using (2.3), we

find the coordinates of the nulls. They are circles of radius $r_{s}( \pm 1) \simeq \sqrt[3]{m / H}$ placed approximately in the coils planes $z= \pm 1$. The magnetic flux throughout the circles is equal to $\Phi_{s}=3 \pi m^{2 / 3} H^{1 / 3}$. Equalizing it to the magnetic flux function $\Phi \simeq \pi H * r^{2}$ in the equatorial plane, we get that the separatrix radius $r_{s}(0)=3^{1 / 2} B_{-}^{1 / 3} / 2^{2 / 3} H^{1 / 3}$ is significantly less there than $r_{0}$.

In the case, where external field is antiparallel to the magnetic dipoles $\left(B_{-} / H<0\right)$, magnetic nulls are placed on plasma axis, while the separatrix surrounds the dipoles themselves and does not cross the equatorial surface.

Thus, in the disk-shaped plasma, inserted into the field of two magnetic dipoles (i.e., coils of small radius), there appears two ring of stability when $H / B_{*}=1.79 \%$. As $H$ increases above the cited value, one of the rings (external one) disappears, while other ring exists up to $H / B_{*} \rightarrow \infty$; however the latter "departs" beyond the separatrix at some value of $H$.

\subsubsection{System of two close coils}

As an example, opposite to the system of two dipoles, we consider the magnetic configuration consisting of two closely placed thin equal coils, inserted into external magnetic field, co-directed to the coils. Once again we take a half of distance between the coils for the measure of length, but now suppose that it is much less than the coils' radius, i.e., $R \gg 1$. Near wire of such coils, their magnetic field once again can be 
considered in plane approximation. We introduce Cartesian coordinate system $\{x, z\}$ with the center, laying at the middle of line which connects parallel currents and with axis $x$ directed along the radius $r=R+x$ of cylindrical system of coordinates. The axis $r=0$ of the coils corresponds to infinitely removed point $x=-\infty$ for the planar approximation $R \gg 1$. Summing fields of linear currents $I$ we find the magnetic field $H$ in the equatorial plane $z$ :

$$
B_{0}=\frac{1}{\pi} B_{\approx} R\left[\delta-\frac{x}{x^{2} \div 1}\right]
$$

where $B_{*}=4 \pi I / c R$ is the field of the coils on their axis in the equatorial plane, and

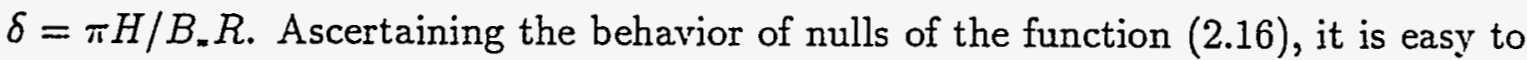
understand that there is a couple of stability rings when $|\delta| \geq 1 / 2$. Apart it, there are two more rings with one of them existing for any value of $\delta$.

The condition for the stability ring to exist (2.11) for the field (2.16) can be reduced to the form

$$
1-\frac{\left(x^{2}-1\right)^{2}}{2\left(x^{2} \div 1\right)} \leq-x\left(x^{2}-3\right)^{2} \delta \leq 1
$$

We first consider the case $\delta>0$, when the external field $H$ is co-directed with the coils' filed on their axis. Then maximum of magnetic field is formed under the coils on their axis. The couple of stability rings, appearing when $\delta \geq 1 / 2$, is placed on both sides from $x=1$ in the region $x>0$, i.e., outside the coils with the current. For $\delta \rightarrow 1 / 2+0$, outer ring of stability is in the region $1+\sqrt{\delta-1 / 2} / \sqrt{3} \leq x \leq 1+\sqrt{\delta-1 / 2}$, and internal ring is placed symmetrically on other side from $x=1$. As $\delta \rightarrow \infty$, outer ring moves to $x=\sqrt{3}$, and internal one moves to $x=+0$, their widths decrease as $1 / \delta$. One more solution of the inequalities (2.17) exists for any $\delta>0$. It corresponds to the most internal ring of stability which, for $\delta \rightarrow+0$, extends from $x=-(2+\sqrt{5})$ formally to $x=-\infty$; in fact it extends to $x \sim-R / l$, where plane approximation breaks. Forth solution corresponds to the most external ring. For $\delta \rightarrow+0$ it occupies wide region $2+\sqrt{5} \leq x \leq+\infty$ but disappears after shrank to zero width at the point $x_{h}=\left(3+2^{4 / 3}+2^{5 / 3}\right)^{1 / 2}=2.95$ when $\delta=x_{h}\left(x_{h}^{2}+3\right) / 3\left(x_{h}^{2}+1\right)^{2}=0.122$. 
Thus, there exist two rings of stability for $0<\delta<0.122<$, one for $0.122<\delta<$ 0.5 , and three for $\delta>0.5$. All three existing rings become very narrow as $\delta \rightarrow \infty$. Their boundaries are fixed by the following inequalities

$$
\begin{aligned}
-\sqrt{3}-1 / 6 \delta & \leq x \leq-\sqrt{3}-1 / 12 \delta \\
1 / 6 \delta & \leq x \leq 1 / 3 \delta \\
\sqrt{3}-1 / 6 \delta & \leq x \leq-\sqrt{3}-1 / 12 \delta
\end{aligned}
$$

Let us now show that in the limit $\delta \rightarrow \infty$ (as well as for $\delta \rightarrow 1 / 2+0$ ) the third ring is separated from the system's axis $x=-\infty$ by separatrix, and, hence, for any $\delta$ can not be used for stabilization of the plasma which occupies near-axis region, i.e., lays inside the separatrix.

Magnetic field nulls lays in the coils' plane when $\delta \gg 1$. Substituting the coordinates of the nulls $x=1 / 2 \delta, z= \pm 1$ into the magnetic flux function

$$
\Phi(x ; z)=\frac{1}{\pi} B_{.} R\left\{x \delta-\frac{1}{4} \ln \left[x^{2}+(z-1)^{2}\right]-\frac{1}{4} \ln \left[x^{2}+(z+1)^{2} \cdot\right\}-\Phi_{0},\right.
$$

we get its value at the separatrix

$$
\Phi_{s}=\frac{1}{\pi} B_{*} R \ln |\mathrm{e} \delta|+\Phi_{0} .
$$

Next, from the equation $\Phi\left(x_{s}, 0\right)=\Phi_{s}$ we find the coordinate of the separatrix in the equatorial plane: $x_{s}=(1 / 2 \delta) \ln |\mathrm{e} \delta|$. Comparing it with (2.18) proves the above made statement.

If $\delta<0$, minimum of magnetic field is formed under the coils. Every results are reproduced from previous ones by means of simultaneous change of signs of $\delta$ and $x$. It appears therefore, that there is by one ring of stability, placed inside the separatrix, for $\delta<-0.5$ and for $-0.122<\delta<0$, but no single ring for $-0.122<\delta<-0.5$. It

' should be mentioned however that the configuration with $\delta<0$ might have no big $\therefore$ value because it has nulls on the axis inside plasma. 


\subsection{Stability of isotropic plasma}

Here we consider isotropic plasma with a specific heat ratio $\gamma$ and introduce the notion for the specific volume

$$
U(\Phi)=\int \frac{d l}{B}
$$

as a function of magnetic flux $\Phi$. The ring of stability in the isoptropic plasma is the region where

$$
U \frac{d}{d \Phi} \Phi \frac{d U}{d \Phi} \leq \gamma \Phi\left(\frac{d U}{d \Phi}\right)^{2} .
$$

Let us ascertain whether the ring of stability appears right after merging of magnetic field nulls as it does in disk-shaped plasma. Published till now results of numerical calculation [7] did not exclude such possibility. They show that in the configuration of two dipoles the ring of stability appears for $H / B_{.} \simeq 2.3 \%$, while nulls merging, as it was mentioned in the Sec. 2.1 , occurs for $H / B_{.} \simeq 1.8 \%$. Little discrepancies between the two values of $H / B_{*}$ could be prescribed to an inaccuracy of numerical calculation.

As before, we can use planar approximation (2.6) near the region of nulls merging.

It was established for the example of disk-shaped plasma that the rings of stability exist only when $H / \beta>0$ and are placed symmetrically on both sides about the line $O O^{\prime}$ which links magnetic field nulls (see Fig. 2.1). Hence, it is sufficient once more to analyze the case $H / \beta>0$ only.

Having noted that $d l / B=d x / B_{x}=d x / 23 x z$, and excluding $z$ by means of the field line equation (2.7) we get from (2.19):

$$
U(\Phi)=\frac{1}{\sqrt{\beta H}} \int_{\xi_{0}}^{\infty} \frac{d \xi}{\sqrt{\xi\left(\xi^{3} / 3+\xi-f\right)}},
$$

where $\xi=|x| / \sqrt{H / \beta}, f=\left|\Phi-\Phi_{0}\right| \sqrt{I I^{3} / \beta}$, and $\xi_{0}$ is a real rout of the equation $\xi^{3} / 3-\xi-f=0$.

The condition (2.20) for the ring of stability to exist under the planar approxima- 
tion comes to the requirement for the minimum of the function

$$
S(f)=U \frac{d^{2} U}{d f^{2}} /\left(\frac{d U}{d f}\right)^{2}
$$

be less than $\gamma$. In the limiting case $f=\infty$ we have $S=4$, while for $f \rightarrow 0$ get $S=-\ln f$. The function (2.22) achieves its minimal value $S_{\min }=2.69$ for $f=0.48$. Thus, in the contrary to the case of disk-shaped plasma, no ring of stability appears at the moment of nulls merging if plasma is isotropic and its specific heat ratio is not fantastically large, i.e., $\gamma<2.69$.

\subsection{Stabilization by sloshing ions}

Let us ascertain now how the effects of non-paraxiality affect stabilization of flute perturbations by sloshing ions. This method of stabilization is based on formation of a piece with relatively large slope of field lines to the system's axis in the region where there pressure peak of an anisotropic plasma is quite big so that 1 !

$$
\frac{\partial}{\partial B} \frac{P(\Phi, B)}{B^{3 / 2}}>0
$$

where $P(\Phi, B)=p_{\sharp}(\Phi, B)+p_{\perp}(\Phi, B)$. The condition (2.23) is obtained with paraxial approximation used, which means that slope angle of field lines is nevertheless small in comparison with right angle.

In order to ascertain how the condition (2.23) is modified after accounting for the effects of non-paraxiality, we consider the magnetic field differing a little from the homogeneous field $H$ :

$$
B_{r}=b_{r}(r, z), \quad B_{r}=H+b_{r}(r, z), \quad B_{\varphi}=0 .
$$

Moreover, we consider that $b_{r, z} \rightarrow 0$ as $z \rightarrow \pm \infty$. When writing out the equation of a field line in such weakly inhomogeneous field, it is useful to distinguish line's small $\therefore$ deviation $\xi \ll r_{0}$ from straight line:

$$
r(z)=r_{0}+\xi\left(r_{0}, z\right)
$$


Sufficient condition for flute modes (large-scale as well as small-scale ones) to be stable in a plasma with steep boundary is $W>0$ where the integral

$$
W=\int d l \frac{\kappa P\left(\Phi_{0}, B\right)}{r B^{2}}
$$

is calculated along the boundary field line. Here $\Phi_{0}$ is the magnetic flux throughout the circle of total plasma radius, $\kappa$ curvature projection onto external normal (directed from the axis) of the field line. Introducing the notation $f(B)=P\left(\Phi_{0}, B\right) / B^{2}$ and keeping the terms of order not higher than first power of $b_{r, z} / H$, we get from (2.26)

$$
W=\int d l \frac{\kappa}{r_{0}}\left[f_{0}(H)-\frac{\xi\left(r_{0}, z\right)}{r_{0}} f_{0}(H) \frac{d f_{0}(H)}{d H}\right] .
$$

Integrating first terms yields zero since $\int d l \kappa=0$ and $B_{r}=0$ at the ends of the interval of integration. As to the remaining terms (already having small parameter), we can substitute there $\kappa=d \xi / d z=b_{r} / H$. Making integration of (2.27) by parts and accounting for

$$
\frac{d}{d z} b_{z}\left(r_{0}, z\right)=-\frac{1}{r_{0}} \frac{\partial}{\partial r_{0}} r_{0} b_{r}\left(r_{0}, z\right)
$$

casts the condition $\mathrm{II}^{-}>0$ into the form

$$
\frac{d}{d B} \frac{P(\Phi, B)}{B^{3 / 2}} \geq \epsilon(\Phi) \frac{P(\Phi, B)}{B^{3 / 2}}
$$

where index ' 0 ' has been omitted, $H$ substituted by $B$, and

$$
\epsilon(\Phi)=r^{4} \frac{d}{d r} \int d z\left(\frac{b_{r}}{r}\right)^{2} / 2 \frac{d}{d r} \int d z\left(r b_{r}\right)^{2} .
$$

In paraxial approximation, the inequality (2.28) coincides with (2.23) since $b_{r} \propto r$ and, hence, $\epsilon=0$.

In the limiting case $\partial \ln \left|b_{r}\right| / \partial \ln r \rightarrow \infty$, which corresponds to "planar" magnetic ficld approximation near the conductors with current, we get $\epsilon=1 / 2$. Hence, instead of (2.23) we have the condition

$$
\frac{\partial}{\partial B} \frac{P(\Phi, B)}{B^{2}}>0
$$


For the latter condition to be satisfied, greater anisotropy is needed than the paraxial region needs.

Now we shall show that the latter conclusion is also valid in the intermediate region between the axis and the conductors. Having $b_{r}$ expanded into Furiet integral of Bessel's functions $I_{1}$ :

$$
b_{r}(r, z)=\int_{-\infty}^{+\infty} d k a_{k} \mathrm{I}_{1}(k r) \mathrm{e}^{i k z}
$$

we find that

$$
\int_{-\infty}^{+\infty} d z b_{r}^{2}(r, z)=2 \pi \int_{-\infty}^{+\infty} d k\left|a_{k}\right|^{2} \mathrm{I}_{1}^{2}(k r)
$$

Since

$$
\frac{d}{d x} \frac{I_{1}^{2}(x)}{x} \geq 0, \quad \frac{d}{d x}\left(x I_{1}(x)\right)^{2} \geq 0,
$$

it is easy to prove that the derivatives of the integrals in both sides of the inequality (2.28) are positive. Hence,

$$
\epsilon(\Phi) \geq \epsilon(0)=0 .
$$

Thus, non-paraxial effects strengthen the requirements for the degree of inisotropy needed for stabilization of flute modes in axisymmetric open mirror devices for plasma confinement.

With rejecting the approximation of quasi-homogeneous field, we now establish obligatory condition of stabilization by sloshing ions for planar magnetic field $\left\{B_{x}(x, z), 0, B_{z}(x, z)\right\}$. Steep boundary plasma in a planar magnetic field is stabile against flute perturbation provided that

$$
W=\int d l \frac{\kappa P\left(\Phi_{0}, B\right)}{B^{2}} .
$$

We adopt the convention to count magnetic flux from plane of symmetry $x=0$, which is analog of the axis if symmetry $r=0$ for the case of an axisymmetric magnetic field, i.e.,

$$
\Phi=\int_{0}^{x} d x B_{z}(x, z)
$$


We shall show that if $P(\Phi, B)=F(\Phi) G(B)$ and

$$
\frac{d}{d B} \frac{G(B)}{B^{2}} \leq 0
$$

for every $B$, then $W / F$ is a monotonically decreasing function of $\Phi$. Since $W=0$ for $\Phi=0$ (i.e., on the system's axis) from this statement it must follow that the opposite inequality

$$
\frac{d}{d B} \frac{G(B)}{B^{2}}>0
$$

is needed to be fulfilled, at least for some values of $B$, for stabilization of flute perturbations.

To prove this, we calculate the derivative $d W / d \Phi$, assuming that magnetic field is vacuum and, hence,

$$
\kappa=\frac{1}{B} \frac{\partial B}{\partial n}=\frac{\partial B}{\partial \Phi},
$$

where $\partial / \partial n$ stands for the derivative over the normal to field line. Bringing in the derivative $\partial / \partial \Phi$ under the integral sign in (2.30) and accounting for $\partial B d l_{l}^{\prime} \partial n=0$, we get

$$
\frac{d}{d \Phi} \frac{W}{F}=\int d l\left[\frac{G}{B^{4}} \frac{\partial^{2} B}{\partial n^{2}}+\left(\frac{\partial B}{\partial n}\right)^{2} \frac{d}{d B} \frac{G}{B^{4}}\right] .
$$

Substituting the relation

$$
\frac{\partial^{2} B}{\partial n^{2}}=\frac{2}{B}\left[\left(\frac{\partial B}{\partial n}\right)^{2}+\left(\frac{\partial B}{\partial l}\right)^{2}\right]-\frac{\partial^{2} B}{\partial l^{2}},
$$

which follows from the equations $\operatorname{div} B=0, \operatorname{rot} B=0$, and integrating the term $\partial^{2} B / \partial l^{2}$ by parts we get finally

$$
\frac{d}{d \Phi} \frac{W}{F}=\int \frac{d l}{B}\left[\left(\frac{\partial B}{\partial n}\right)^{2}+\left(\frac{\partial B}{\partial l}\right)^{2}\right] \frac{d}{d B} \frac{G}{B^{2}},
$$

which ends the proof. 


\section{Bibliography}

[1] Timofeev A.V.// JETPh Letters. 1979. V.29. P.227.

[2] Logan B.G.// Comments on Plasma Phys. and Contr. Fusion. 1980. V.6. P.199.

[3] Hinton F.L., Rosenbluth M.N.// Nuclear Fusion. 1982. V.22. P.1547.

[4] Kotelnikov I.A., Roslyakov G.V., Ryutov D.D.// Fizika plazmy. 1987. V.13. P.403.

[5] Arsenin V.V.// JETPh Letters. 1983. V.37. P.534.

[6] Kesner J.// Nuclear Fusion. 1985. V.25. P.275.

[7] Ryutov D.D., Stupakov G.V.// Fizika plasmy. 1986. V.12. P.1411. 


\section{Chapter 3}

\section{Experiments on the GDT facility}

Main Contrubutors: V.Anikeev, P.A.Bagrianskii, P.A.Deichuli, A.N.Karpushov,

V.N.Khudik, A.Ivanov, A.I.Rogozin, T.V.Salikova

3.1 Measurements of a plasma equilibrium response to external multipole fields in an ax. isymmetric gas-dynamic trap

V.Anikeev, P.A.Bagrianskii, V.N.Khudik, A.Ivanov, T.V.Salikova

\subsubsection{Introduction}

In this paper we report on the results of studies of a plasma equilibrium in the gasdynamic trap in the presence of small non-axisymmetric disturbances of the main magnetic field. These studies were initiated by experimental observations on the plasma behavior during its decay in various configuration of the magnetic field. Re- cent studies of a gun-produced plasma decay in the GDT facility have shown that - violation of the MHD-stability criterion results in growth of large-scale flute-like per- 


\begin{tabular}{|c|c|}
\hline Parameters & Value \\
\hline Mirror-Mirror & $7 \mathrm{~m}$ \\
Diameter of Central & \\
Cell Vacuum Chamber & $1 \mathrm{~m}$ \\
Magnetic Field & $0.22 \mathrm{~T} / 16 \mathrm{~T}$ \\
Coils Current Duration & $\sim 100 \mathrm{~ms}$ \\
Base Pressure & $5 \times 10^{-7} \mathrm{Torr}$ \\
Central Cell & \\
Plasma Density & $0.5-1 \times 10^{14} \mathrm{~cm}^{-3}$ \\
Electron Temperature & up to $40 \mathrm{eV}$ \\
\hline
\end{tabular}

Table 3.1: Parameters of the GDT facility.

turbations. When driven unstable these perturbations cause fast plasma losses across the magnetic field :1?. During these experiments it was found that the plasma behavior, in particular, in equilibrium state exhibited a significant role of non-axisymmetric disturbances of magnetic field. This gave raise to experimental efforts to qualify these effects and, if possible, find the ways of their controlling. The experimental layout is schematically depicted in Fig.3.1.

The GDT experiment consists of an axisymmetric central cell and two outboard MHD-anchors attached from both ends. Axisymmetric magnetic field has a strength of $0.15-0.22 \mathrm{~T}$ at the midplane and up to $16 \mathrm{~T}$ at magnetic mirrors. The field was produced by a set of axisymmetric coils installed on the vacuum chamber. Main parameters of the GDT facility are summarized in the Tabl.3.1. More details on the facility and key objectives of the experimental program it was build for, are given in $[2]$.

The plasma build-up in the trap was performed by a plasma gun located beyond the magnetic mirror in one of the end tanks. During the gun operation plasma 
remained macroscopically stable, within certain limits, even if the instability threshold defined by the pressure-weighted curvature criterion [3]was exceeded. This stability was attributed to the electric contact of plasma in the central cell with the plasma inside the gun where it is supposed to be highly conductive across the magnetic field. The peak plasma density reached during a typical shot for $3 \mathrm{~ms}$ gun operation was $\sim 6 \times 10^{13} \mathrm{~cm}^{-3}$ with electron temperature $5-10 \mathrm{eV}$.

As it was previously reported [1] after the gun was turned off, the plasma behavior and parameters of the decay became sensitive to the areraged curvature of the field lines in the trap. In an unstable decay, the azimuthal spectrum of driven perturbations was found be dominated by a rigid-shift mode of $m=1$. Further experiments [4] have shown that measured correlation between the growthrates of unstable azimuthal modes are consistent with the theory including finite Larmor radius effects [5].

These effects are significant for standard conditions of the GDT experiments even if any auxiliary heating (ICRF or neutral beams) was not applied to increase the plasma temperature over its initial value of $\sim \overline{\mathfrak{j}}-10 \mathrm{e} V$ just after the gun off.

Temporal development of the flute modes were observed with the use of Langmiur probes located near the magnetic mirrors at various azimuthal positions. Design of the probe array and parameters of the hardware were those that allow to measure amplitudes and phases of the modes up to $\mathrm{m}=12$ in $100 \mathrm{kHz}$ frequency band. Plasma column offset and radial width were measured by making use of linear probe arrays located in the central cell and inside the expander.

In the stable decays, plasma column after the gun turned off had a chaotic initial offset from the geometrical axis of the trap. Subsequently, during $\sim 300-500 \mu \mathrm{sec}$, the plasma ivolved to a position that was almost the same for different shots. The motion had a form of shifting into the final position that further remained invariable during the decay $(\sim 2 \mathrm{~ms})$ or it appeared as a damped oscillation about this position. A few samples of trajectories of center of the plasma column during the relaxation are 
shown in the Fig.3.2. As it easily seen from the figure the final position of the plasma centroid is shifted considerably from the geometrical axis of the machine.

In [6] the influence of external non-axisymmetric disturbances on a plasma equilibrium in a long axisymmetric open trap was treated in approximation of ideal MHDtheory. It was found that the plasma should respond to dipole disturbances by off-axis shifting. Higher order multipole disturbances should cause more complicated perturbations of pressure profile with corresponding azimuthal number. We attributed the plasma offset observed in our experiments to uncontrollable dipole disturbances of the magnetic field. Rough estimations have indicated a few gauss transverse field would be sufficient for that. In particular, residual dipole disturbances of the main axisymmetric field may result from misalignments of the coils, existing of soft-ironmade parts near the device, etc. Because of the pulse character of the main magnetic field, screening currents in metallic structures of the walls of the building also provide considerable contribution to the distortions. Note that the unstable decays also exhibited a preferable direction of the centroid motion during the decays.(see Fig.3.3).

Completely avoid distortions of the axisymmetric magnetic configuration is practically impossible. We therefore concluded that it is very important to develop reliable control system of a plasma equilibrium position and shape which will be able to reduce these distortions to acceptable level. Furthermore, this system, of course if it has a proper frequency response, can be used to stabilize large scale flute perturbations. In this report we studied the possibility to control the plasma equilibrium by the use of coils that produce a small transverse magnetic fields in the regions of the plasma expansion beyond the mirrors. As it was first mentioned in :6], because of the fact that the main magnetic field here is quite small, the external field applied in these regions altering the equilibrium to a maximum degree. 


\subsubsection{Estimates of the equilibrium parameters in the pres- ence of multipole disturbances.}

A plasma equilibrium in an axisymmetric gas-dynamic trap with multipole external disturbances of the magnetic field was first discussed theoretically in [7]. The equilibrium was treated in an approximation of ideal magneto-hydrodynamic. To facilitate the discussion of our experiments we display the main relationships following mainly to the results of $[6,7]$. In case when a small dipole field is applied, the components of the magnetic field can be written in the form ( using a long thin approximation): $H_{z}=H(z), H_{x}=\delta H_{x}(z)-\frac{x}{2} H^{\prime}(z)$, where $\mathrm{H}(z)$ - is unperturbed on-axis magnetic field. It follows from the results of [7] that at a midplane, surfaces of a constant plasma pressure are to be enclosed circles:

$$
\left(X+\frac{A}{C}\right)^{2}+\left(Y+\frac{B}{C}\right)^{2}=\text { const }
$$

Here $\mathrm{X}$ and $\mathrm{Y}$-are coordinates of a point at the midplane,

$$
\begin{aligned}
& A=\int_{-\ell}^{\ell} \frac{F(H) d s}{H^{3 / 2}} \frac{d^{2}}{d z^{2}} \delta x \\
& B=\int_{-\ell}^{\ell} \frac{F(H) d s}{H^{3 / 2}} \frac{d^{2}}{d z^{2}} \delta y \\
& =\int_{-\ell}^{\ell} \frac{F(H) d s}{H^{3 / 2}} \frac{d^{2}}{d z^{2}} R^{-1 / 2}
\end{aligned}
$$

function $F(H)$ describes pressure distribution along magnetic field lines in the gasdynamic trap[8] :

$P_{\perp}+P_{\mathrm{i}}=2 P(X, Y) F(H), \mathrm{R}$ - current mirror ratio , functions $\delta x=R(z)^{-1 / 2} \int_{-\ell}^{\ell} R(z)^{1 / 2} \frac{\varepsilon H_{z}}{H(z)} d z$ $\delta y=R(z)^{-1 / 2} \int_{-\varepsilon}^{\ell} R(z)^{1 / 2} \frac{\varepsilon H_{y}}{H(z)} d z$ were the integrands comprise corresponding components of the perturbations of the field- $\delta H_{x}, \delta H_{y}$.

If, simultaneously; a quadrupole disturbance of the form $\delta H_{x}=-2 b(z) x ; \delta H_{y}=$ $\left.+2 b(z) y: \delta H_{z}=-\left(x^{2}-y^{2}\right) \frac{d g}{d z}\right)$ is applicd, it makes the surfaces of a constant pressurc be ellipsoids:

$$
\frac{\left(X+\frac{A}{C}\right)^{2}}{\hat{C}^{2}}+\frac{\left(Y+\frac{B}{C}\right)^{2}}{C^{2}}=\text { const }
$$


with coordinates of a center: $\left[-\frac{A}{C} ;-\frac{B}{\tilde{C}}\right]$ and ellipticity of $\frac{a_{x}}{b_{y}}=\frac{\hat{C}}{C}$.

Parameters of the ellipsoids are functions of the following integrals:

$$
\begin{gathered}
A=\int_{-\ell}^{\ell} \frac{d s F(H) R^{1 / 2} E^{1 / 2}}{H^{2}} \frac{d^{2}}{d z^{2}} \delta x \\
B=\int_{-\ell}^{\ell} \frac{d s F(H) R^{1 / 2} E^{-1 / 2}}{H^{2}} \frac{d^{2}}{d z^{2}} \delta y \\
C=\int_{-\ell}^{\ell} \frac{d s F(H) R^{1 / 2} E^{1 / 2}}{H^{2}} \frac{d^{2}}{d z^{2}} R^{-1 / 2} E^{-1 / 2} \\
\hat{C}=\int_{-\varepsilon}^{\ell} \frac{d s F(H) R^{1 / 2} E^{-1 / 2}}{H^{2}} \frac{d^{2}}{d z^{2}} R^{-1 / 2} E^{1 / 2}
\end{gathered}
$$

where $E(z)=\exp \left(4 \int_{0}^{z} \frac{b(z)}{H(z)} d z\right)$ is a function of the $\mathrm{b}(z)$, that describes an amplitude of the quadrupole perturbation of the field.

Value of the integrals which define off-axis shift of the entire plasma column and its ellipticity, strongly depends on a choice of upper limit that corresponds to the points near end walls where the magnetic field is small. It is quite obvious that response of the plasma column is determined mainly by a region at the radial profile where pressure gradient has its maximum. According to this consideration, while integrating along the axis we searched for the parameters at these field lines at the same z-positions. We fixed the upper limit in $z$ by a condition that one of the two imposed limitation will be broken at a field line corresponding to a maximum of the pressure gradient: $\kappa \rho \leq \kappa \rho_{\text {crit }}, \beta \leq 1$. For our experimental conditions, practically the former limitation was violated. The value of $\kappa \rho_{c r i t}$ in calculations was varied from 0.1 to 0.5 .

It is worth noting that stability properties of the plasma can be judged quantitatively from the data on the plasma offset as a function of amplitude of applied dipole perturbation. The C-integral entering the Eq.1 represents. with an accuracy of multiplying on a nonessential factor, the pressure weighted curvature. Exactly the same expression enters the MHD-stability criterion [3] for the localized modes with $m \gg 1$. Of course, we consider a paraxial approximation to be valid. 
Let split the stability integral into two parts one of that corresponds to segment of the field line in the central cell and another to that in the end cells. The stability criterion is then can be reformulated in terms of a "safety factor" $Q$ which is defined as absolute value of the ratio of these integrals. The plasma will be stable against curvature-driven flute localized modes when $Q \geq 1$. Similarly, the safety factor can be defined for arbitrary radial and azimuthal modes. In our previous experimentsi9], the Q-value measured for the gun-produced plasma in the GDT facility appeared be somewhat less than calculated for $\mathrm{m}=1$ mode with $\kappa \rho_{\text {crit }}=0.5$.

Measurements of the plasma off-axis shift under dipole disturbance externally applied inside the expander, can provide one with the data on the safety factor for high-m localized modes. This value then can be used to estimate $Q$ for large-scale modes which control the plasma life time when driven unstable. Within the frames of the model used in $[9,10,11]$ for analysis of the experimental data one is able to obtain independent estimate of $k \rho_{c r i t}$, which define upper limit in $\mathrm{C}$-integral. This approach suggests, of course, that parameters of the plasma flow in the expander is correctly covered by either izothcrmal or adiabatic model $i 10$.

A comparison to the experimental data on electrostatic potentials in expander show, that these models are reasonably accurate only for high electron temperature in the central cell $99^{\circ}$. Nevertheless, this approach to estimate the parameter of $\kappa \rho_{\text {crit }}$ seems to be important.

\subsubsection{Results of measurements.}

Dipole perturbations.

Experimental layout and locations of the main diagnostics used are shown in Fig.3.4. Dipole perturbation in the expander was produced by a pair of coils with radius of 0.81 installed at the distance between them of 2.1 (not shown in the figurc). The magnetic field generated by the coils was practically homogeneous over the region in expander occupied by the plasma. Fig.3.5 shows experimentally measured shift of 
the plasma centroid as a function of transverse field amplitude in the expander.

Also shown in the Fig.3.5 are calculated curves for various values of $\kappa \rho_{\text {crit }}$ in the case of adiabatical plasma flow in expander. The curve corresponding to $\kappa \rho_{\text {crit }}=0.3$. gives the best fit to experimental data. Sensitivity of the calculated offset to the limitation imposed on $\kappa \rho_{\text {crit }}$ is illustrated by the curves with $\kappa \rho_{\text {crit }}=0.1 ; 0.5$ (curves,).

Quadrupole perturbation.

Quadrupole perturbations in the expander were generated by four properly connected coils of $0.5 \mathrm{~m}$ in diameter (Fig.3.4). Ellipticity of the plasma column was measured by a linear array of Langmiur probes (6) located inside the central cell and by $8 \mathrm{~mm}$-interferometer (8) located just beyond the mirror throat in expander. To obtain the ellipticity from interferometer data, we used values of $\langle N L\rangle$ measured along the perpendicular plasma diameters.

On-axis amplitude of the perturbation, determined by the function $b(z)$, signifcantly changed inside the expander. Fig.3.6 shows experimentally measured ellipticity as a function of $b(z)$-value directly in the $z$-point where disturbing coils were located. We obscrved the expected ellipticity, calculated for $\kappa \rho_{\text {crit }}=0.3$, being approximately 3-4 times larger then the measured values.

Possible reasons for that will be discussed in the next section.

\subsubsection{Discussion.}

As it was already mentioned, in our previous experiments on the MHD-stability limits in the gas-dynamic trap, we have found a contribution of the expanders to the stability criterion be considerable less than calculated for $\kappa \rho_{\text {crit }}=0.5$. Present experiments also indicate that $\kappa \rho_{\text {crit }}=0.3$ is a more appropriate value to be used. Thus it can be concluded that the safety factor inferred from the data on the equilibrium response to dipole disturbance gives a reasonable estimation.

The origin of the difference between expected and measured responses of the plasma equilibrium to quadrupole disturbance is now not well understood. The ideal 
MHD-theory predicts the response to be inversely proportional to slightly modified stability criterion of localized modes $[6,7]$. Ryutov [12] has pointed out that where may be several pitfalls. Specifically, he suggested that the effects of finite ion Larmor radius may qualitatively change the response as they prevent a plasma be distorted with azimuthal numbers $m \geq 1$. The same should be valid if the entire plasma column will forced to rotate by a transverse electrical field. Further theoretical considerations and experiments are needed to provide quantitative answers. Comparison of measured response to quadrupole perturbations apparently indicated that there should be a cause of its significant reduction comparing to ideal MIHD-theory.

The majority of data were taken when the magnetic field in the end tank was of the expander's configuration. During these experiments the cusp coils have been already installed on the GDT. Thus, properly reconnecting outer and inner coils of the end tank we were able to choose the cusp or expander for use in a certain series of shots. A distinct feature of the cusp-anchored gas-dynamic trap is the singularity of a

specific volume of flux tubes $\int \frac{d l}{B}$ near the axis. A theory that relevant to this case [13] deduces the equilibrium response quite different comparing to that for the trap with an expander end cell. The ellipticity vs amplitude of a quidrupole disturbance in the cusp is presented in Fig.3.7. The fitting curve for the experimental data reasonably agree with our theoretical estimates.

\title{
3.2 A neutral beam probe for measurements of density fluctuations in the GDT experiment.
}

\author{
A.A.Ivanov, A.I.Rogozin
}

A neutral beam prube was developed to measure density profile and fluctuations in the central cell of the GDT. This diagnostics has been already used in the initial stage of the GDT experimental program [14]. Yet it was found that a current and an energy $(15-30 \mathrm{kV})$ of the ion source used are too small to obtain reasonable data with 
powerful neutral beam heating. Notwithstanding the diagnostics was located beyond the turning point of the sloshing ions, scattered particles provided too large noise signal to surface-barrier detectors. This was very difficalt to avoid since the energy of scattered particles were of the same order as in the diagnostic beam $(\sim 10-15 \mathrm{kel})$. Newly developed diagnostics has increased capabilities due to higher accelerating voltage and the beam current. Fig.3.8 is a schematic diagram of the major components of the system designed for GDT. The neutral beam probe incorporates a deuterium neutral beam and a secondary ion detectors. The detectors are collimated to see the ions borning from the beam particles on small segments of the beam trajectory. The collisional ionization rate of the beam atoms is almost independent on plasma temperature. Thus, the density profile can be deduced from detector currents data and knowledge of the large (with a scale of $\sim 1 \mathrm{~cm}$ ) density fluctuations along the beam. Main parameters of the diagnostics equipment is listed in the Tabl.3.2.

\begin{tabular}{|c|c|}
\hline Parameters & \\
\hline Species & $D^{+}, H^{+}$ \\
Beam energy & up to $60 \mathrm{kV}$ \\
Beam current & $0.1-2.2 \mathrm{~mA}$ \\
Initial beam diameter & $10 \mathrm{~mm}$ \\
Beam divergence & $\sim 1 \mathrm{deg}$. \\
Spatial resolution (achieved in GDT) & $\sim 1 \mathrm{~cm}$ \\
Time resolution (achieved in GDT) & $\sim 20 \mu \mathrm{sec}$ \\
RF-power(27MHz) required to ion source & $200 \mathrm{~W}$ \\
\hline
\end{tabular}

Table 3.2: Parameters of the source and power supply system.

The parameters presented at the Tabl.3.2 are those achieved during tests before installing on the GDT midplane. Initial attempts to operate on GDT have shown that residual detector currents during neutral beam heating are small enough allowing to 
start the measurements.

\title{
3.3 Stability properties of a cusp-anchored gas- dynamic trap.
}

\author{
V.Anikeev, P.A.Bagrianskii, P.A.Deichuli, A.N.Karpushov, \\ A.A.Ivanov, T.V.Salikova, Yu.A.Tsidulko
}

\subsubsection{Introduction.}

Theory predicts that it is possible to increase the safety factor over that for a single expander if one uses a cusp end-cell. The axial confinement time of a collisional plasma in a cusp is substantially large then that for the expander. This circumstance can be exploited to obtain more dense plasma for the same inward flux from the central ccll. A pressure-weighted curvature of the field lines corresponding to a maximum density gradient at the midplane is inversely proportional to a magnetic pressure which is much higher in the cusp then in the expander. However, increased density in the cusp overpowers the influence of this unfavorable factor. Simple particle balance model was developed to estimate the plasma parameters in the cusp. It was assumed that the density on a field line is determined by a balance of collisional flows through a point and an outer ring cusps. We also assumed the entire volume of the cusp filled by the plasma of a constant density. To aroid the diocotron instability driven by a high speed ion drift in the layer of the ring cusp the limitation was applied that the layer width should be large then two ion Larmor radii. Additional coils set were designed to generate optimal cusp configuration. In early 1992 the GDT facility was fitted by this coils set. Fig.3.9 shows a schematic diagram of the cusp end-cell. Main parameters of the cusp are summarized in the Tabl.3.3.

The estimation of safety factor included in the Tabl.3.3 refers to the case of $50 \mathrm{eV}$ electron temperature. 


\begin{tabular}{|c|c|}
\hline Parameters & \\
\hline Ring Cusp Field & up to 1.76T \\
Minimum Field * & $0.04 \mathrm{~T}$ \\
Plasma $\beta$ & 0.1 \\
Calculated Safety Factor & 5 \\
Layer Width in Ring & \\
Cusp & $0.12 \mathrm{~cm}$ \\
Ring Cusp Radius & $53.5 \mathrm{~cm}$ \\
Ion Larmor Radius & \\
in the Ring Cusp & $0.06 \mathrm{~cm}$ \\
\hline
\end{tabular}

Table 3.3: Cusp Parameters.

\subsubsection{Experiments with Gun-Produced Plasma.}

A hydrogen-fed plasma gun located in the opposite expander was used for plasma build-up in the trap. The gun was normally operated within $3 \mathrm{~ms}$. The initial plasma density within the range of $\bar{\jmath}-10 \times 10^{13} \mathrm{~cm}^{-3}$ and electron temperature $5-10 \mathrm{eV}$ can be established. The radial plasma profile in the cusp in the vicinity of the mirror throat is shown on the Fig.3.10. Local radial coordinates shown on the Fig.3.10 are those that were mapped on to the midplane. The density was measured by a movable triple probe from shot to shot. Existence of a cavity in the profile can be explained by the fact that bundles of the field lines near the cusp axis have a larger rolume to fill by the plasma flow from the central cell. We performed calculation runs to verify this assumption using by experimentally measured values of plasma density and temperature in the central cell during the plasma build-up. The results were found be quantitatively consistent with the data. They are also presented in the Fig.3.10. Outer half of the cusp was almost empty with the exception of the near-axis region. Possibly it was due to the plasma penetration through the null 
of the cusp. The unfavorable consequence of the cavity existence is that since the local contribution to stability integral is determined by the local density gradient (particularly by its sign), the safety factor for global MHDD-modes will be decreased.

Fig.3.11 shows plasma profile in the ring cusp. The width of the profile was in a good agreement with the measurements near the magnetic mirror. This indicates the absence of significant plasma losses in the layer. We were able to change plasma pressure in the cusp and its contribution to stability criterion by varying of the mirror ratio in the central cell. Similar procedure was used previously for measurements of safety factor for the GDT with the expander[9]. The plasma life time vs mirror ratio from the central cell to the cusp is plotted in the Fig.3.12. In contrast to our expectations, above the mirror ratio of $\sim 30$ plasma lifetime rapidly falls down indicating enhanced plasma losses. All diagnostics also have shown a development of large scale perturbations in the central cell which would cause these losses. It was observed that the plasma motions in the central cell and in the cusp were not correlated. Plasma position in the cusp was absolutely insensitive to a large amplitude shift of the plasma column in the central cell. We attributed this observation to the drive of the resistive ballooning instability [15, that had to occur for small plasma temperatures. Our study of the plasma heated by neutral beams demonstrates the importance of the electron temperature. There are some indications of a trend for the correlations to raise with $T_{e}$ in qualitative agreement with the theory[15]. Since we were not satisfied with the existence of the cavity on the density profile in the cusp we tried to fill it by making use of a plasma gun which fired directly into the cusp. Density profile and temporal evolution of on-axis plasma density which were measured with additional gun on is shown in Fig.3.13. ('urre labeled a) in the Fig.3.13 illustrates a time history of the on-axis density during the additional gun operation. 


\subsubsection{Experiments with Neutral Beam Heating.}

As the gun-produced plasma was observed to be unstable we were continuing experiments with the neutral beam heating. Temporal dependence of absorbed power from the neutral beams operated with $15 \mathrm{kV}$ energy is presented in Fig.3.14. Also shown in the Fig.3.14 are total power of charge exchange losses, power transferred to plasma electrons by the drag of the sloshing ions and a growthrate of the sloshing ion's energy content. Electron temperature of the plasma during the heating and energy content of sloshing ions are shown in the Fig.3.15;3.16.

Multichord attenuation data obtained from the main neutral beams are presented in Fig.3.17 for the shots with the cusp coils on and off. In the latter case, the plasma was strongly unstable. Rapid plasma perturbations with characteristic scales of order of the plasma radius were observed. In the former case, in contrast, the plasma profile was well defined although fast motions of the plasma were also monitored. Plasma density at the periphery was even increasing during the heating which we attributed to a gas release from the walls impinged by the beams. Unfortunately, because of the fast changes of the plasma parameters during the decay with the neutral beam heating and a significant shot-to-short variability we were not able accurately measure the safety factor of the cusp-anchored gas-dynamic trap. Nevertheless, the bulk plasma temperature in the range of $30-40 \mathrm{eV}$ and maximal density of the sloshing ions of order of $\sim 1 \times 10^{12} \mathrm{~cm}^{-3}$ were obtained. Thus we concluded that the plasma is globally stable at least for the large scale flute-like modes. Temporal behavior of the safety factor during the heating was calculated with the use of the plasma parameters measured in the central cell and in the cusp( Fig.3.18). For the hollow plasma profile the safety factor for the entire plasma accounting contribution of the sloshing ions appeared to be above the instability threshold while for the filled profile it was favorable throughout a decay (curve b)). We suspect that the plasma behavior in these experiments may has

a certain concern to the theory described in the first two chapters of present report. It is very likely that effects of magnetic drift of the sloshing ions and an interaction 
with a limiter result in significant difference of stability properties expected from ideal MHD-theory. 


\section{Bibliography}

[1] P.A.Bagryanskii, A.A.Ivanov, V.V.Klesov et al., Proc. XI IAEA Conf. on Plasma Phys. and Controlled. Nucl. Fusion Res., v.3, p.467, Vienna(1987)

[2] P.A.Bagryanskii, A.A.Ivanov, V.V.Klesov et al. Phys. of Mirrors, Reversed Field Pinches and Compact Tori, Proc. of the Course and Workshop, Varenna, Italy, r.2, p.635, Editrice Compositori,Bologna(1987).

3. M.X.Rosenbluth, C.L. Longmire, Ann. Phys.,1,20(1957).

I. P.A.Bagryansky,A.A.Iranov,S.V.Kuzmin,T.V.Salikora, and A.T.Anikeer, Plasma Phys.and Controlled Fusion 34,1185(1992).

5.: M.N.Rosenbluth,N.F.Kroll,N.Rostoker, Nucl.Fus.,suppl., part 1(1962)143.

[6] I.A.Kotel'nikov, preprint INP \#83-36(1983)(in Russian).

[7] I.A.Kotel'nokov, $\mathrm{PhD}$ thesis,INP, Novosibirsk(1985)(in Russian).

18: V.P.Nagorny, D.D.Ryutov, G.V.Stupakov, Nucl.Fusion.24,1421(1984).

9.: P.A.Bagryansky, A.A.Iranov, V.V.Klyosov, I.A.Kotel'nikov, Yu.I.Krasnikov, G.V.Roslyakov, Yu.A.Tsidulko, R.A.Breun, A.W..lolvik, T.A.Casper, Extended Synopses of XIII IAEA Conf on Plasma Phys.and Controlled. Nucl. Fusion Research.,p.126,IAEA, Vienna(1990), preprint INP \#9168(1991). 
[10] V.V.Mirnov, and D.D.Ryutov, Voprosy Atomnoj Nauki i Tekhniki -Termoyadernyj Sintez, vol.1(1980)57(in Russian).

[11] I.A.Kotel'nikov,V.V.Mirnov,V.P.Nagornyj, and D.D.Ryutov, Proc. X IAEA Conf on Plasma Phys. and Controlled.Nucl.Fusion Res., v.2,p.309, Vienna(1985)

[12] D.D.Ryutov, private communication(1992.)

[13] A.I.Shetnikov, preprint INP \#86-46(1986)(in Russian).

[14] P.A.Bagrianskii et al., Proc. of XIX Int. Conf. on Phenomena in Ion. Gases, Belgrade, p.832-833, (1989).

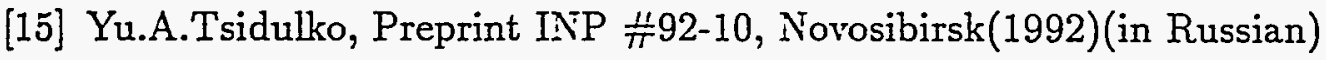




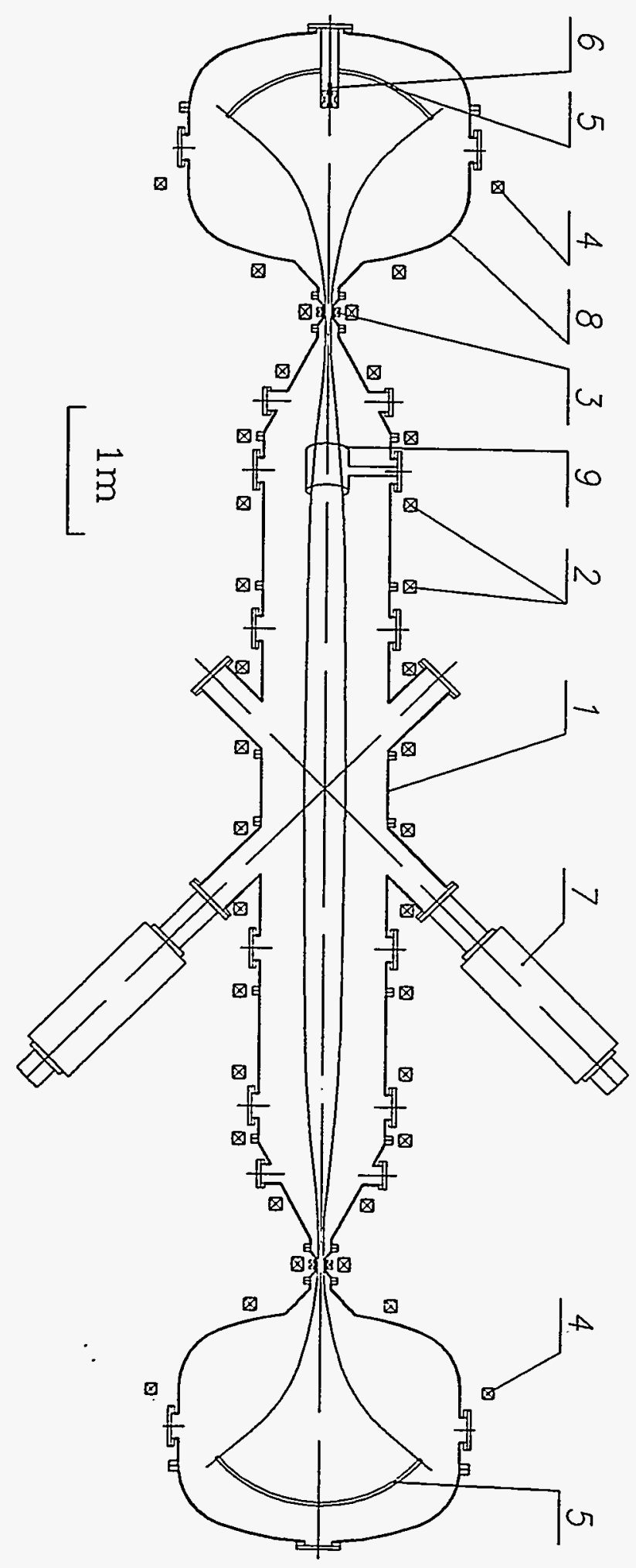

Figure 3.1: The GDT layout. 


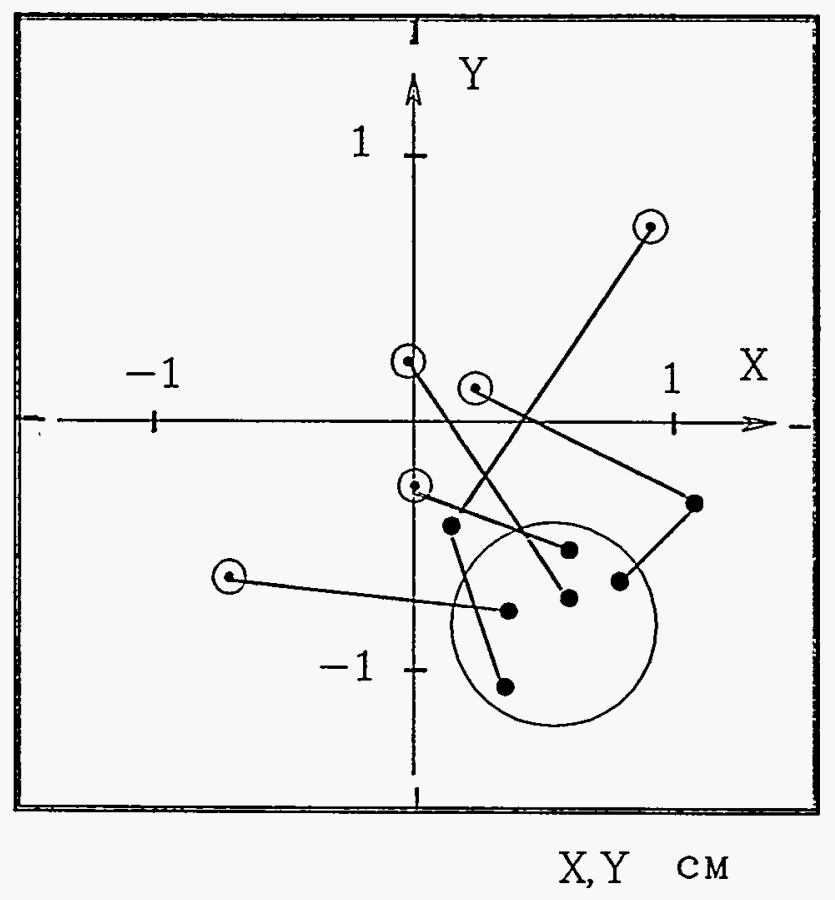

Figure 3.2: Plasma centroid motion during stable decays. 


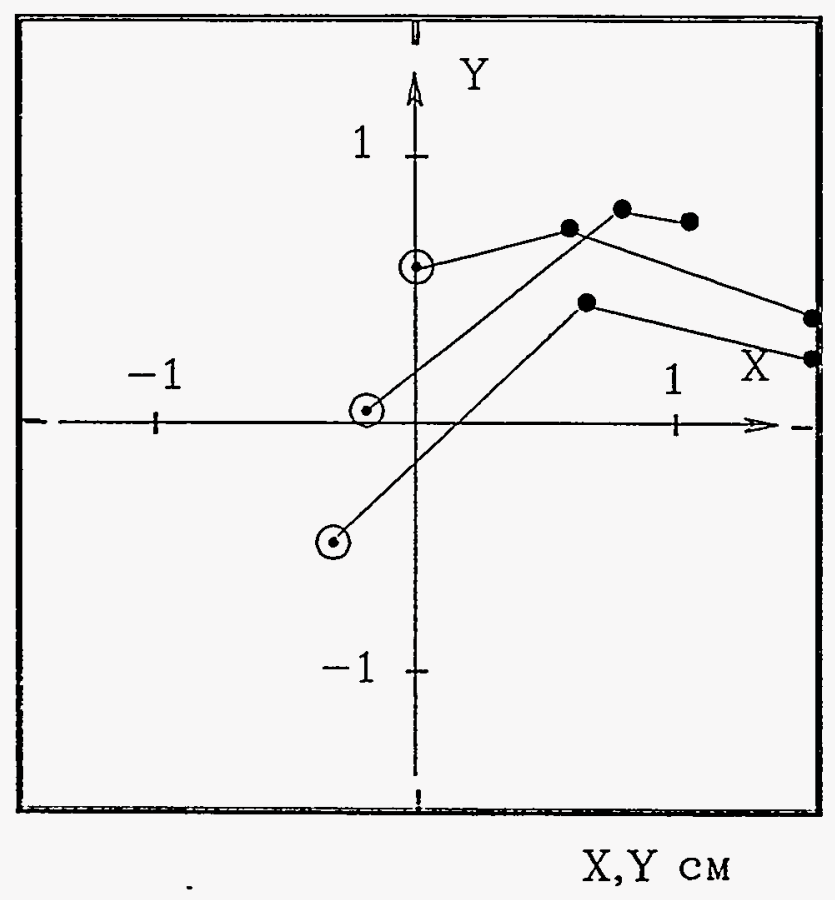

Figure 3.3: Centroid motion during unstable decays. 


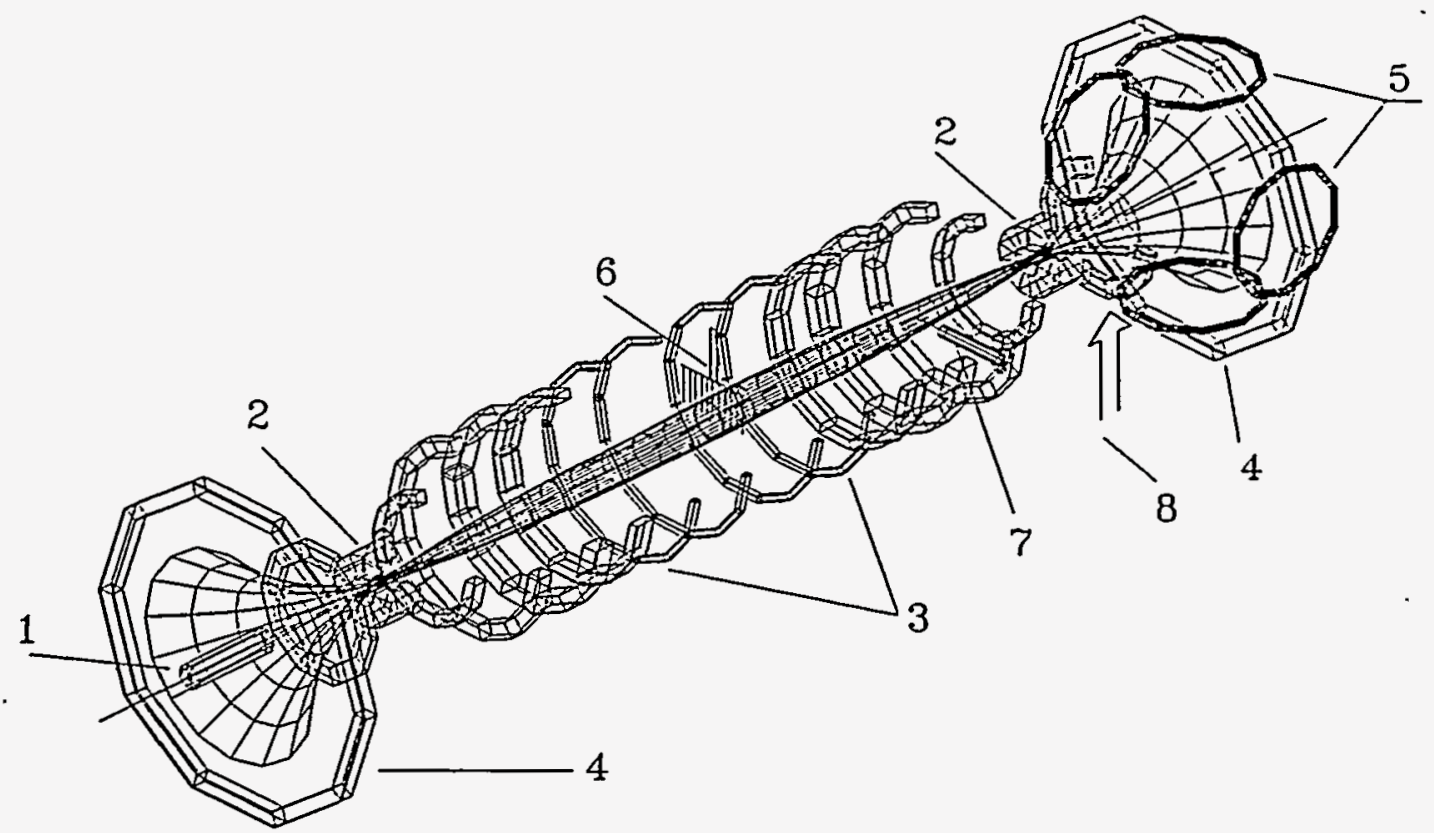

Figure 3.4: Experimental layout and diagnostics.

1. Plasma gun; 2. Mirror coils; 3. Central cell coils;

4. Expander coils; 5. Disturbing coils; 6. Linear probe array; 7. Movable triple probe; 8 . $8 \mathrm{~mm}$-interferometer. 


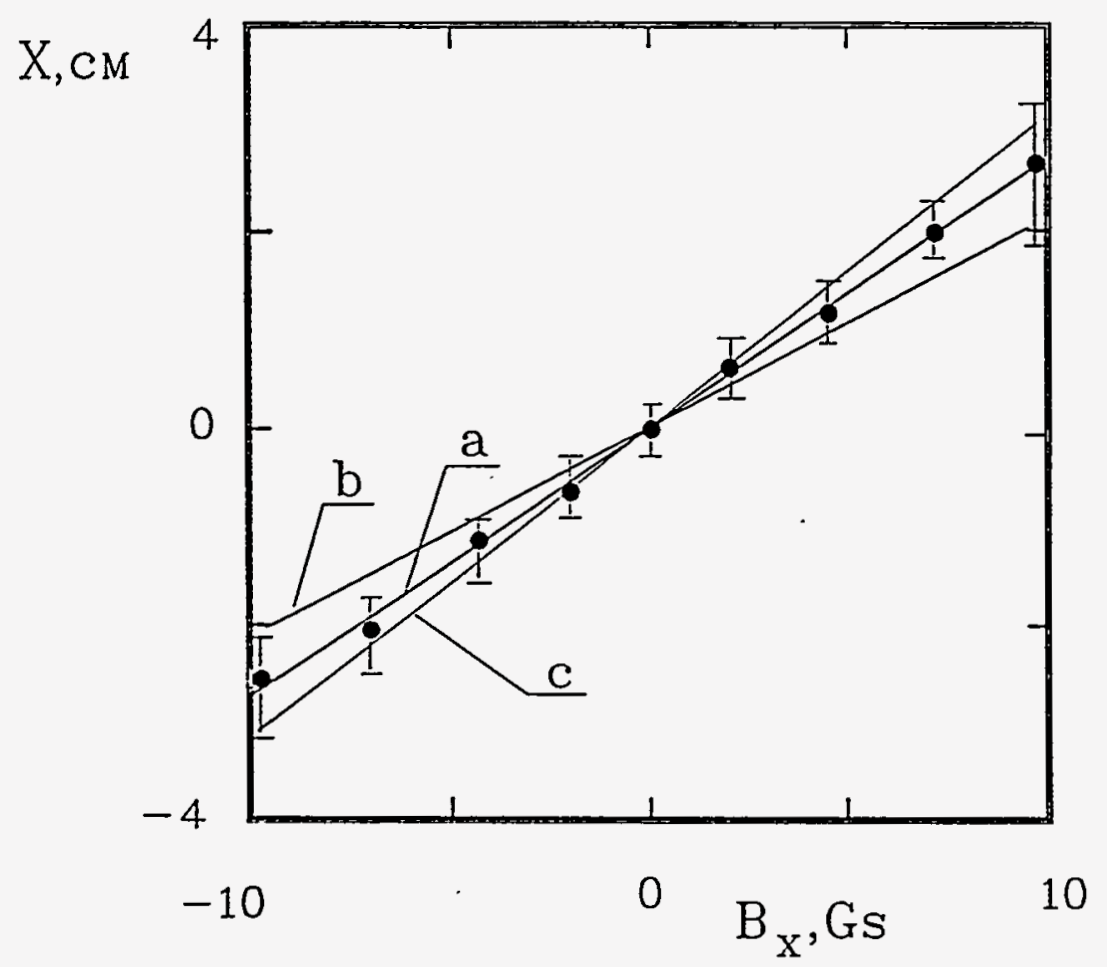

Figure 3.5: Plasma offset vs amplitude of dipole disturbance 


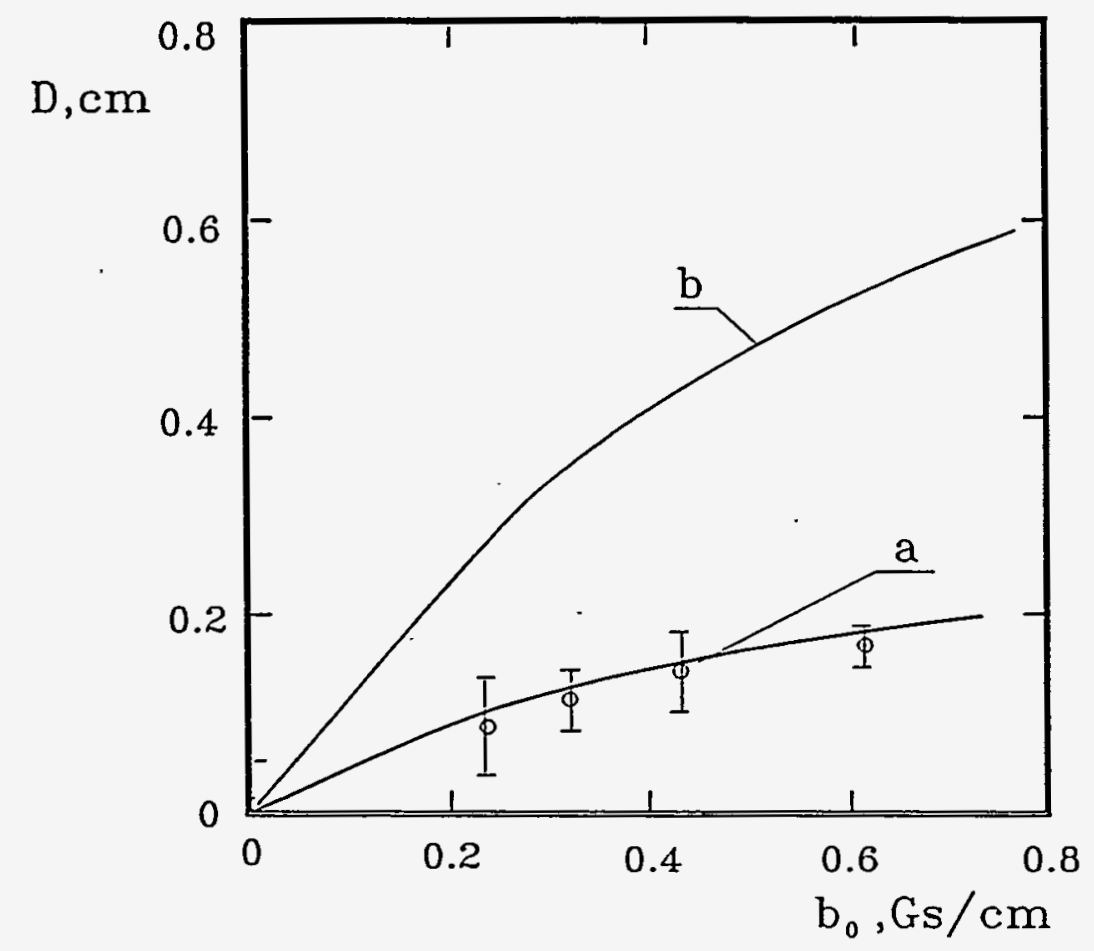

Figure 3.6: Elliptisity vs amplitude of quadrupole disturbance. 


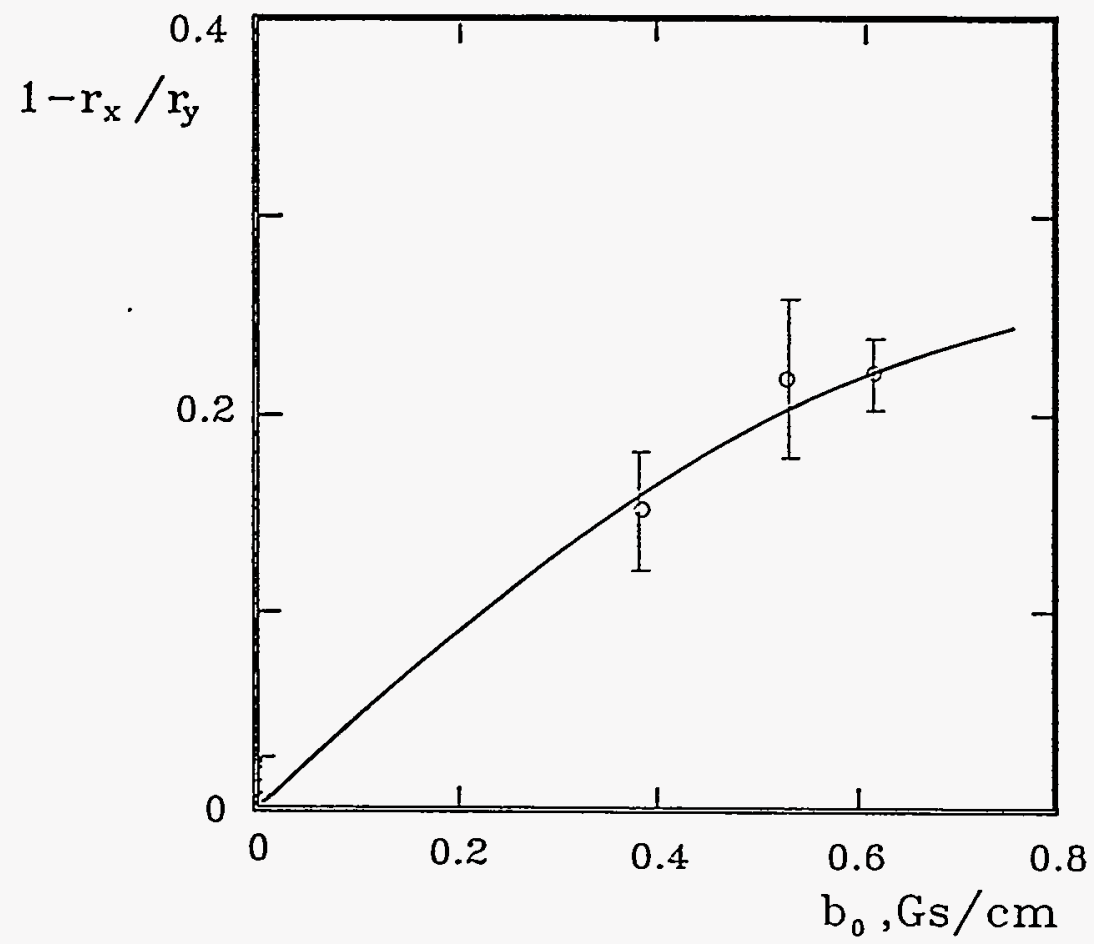

Figure 3.7: Elliptisity vs amplitude of quadrupole disturbance in the cusp. 


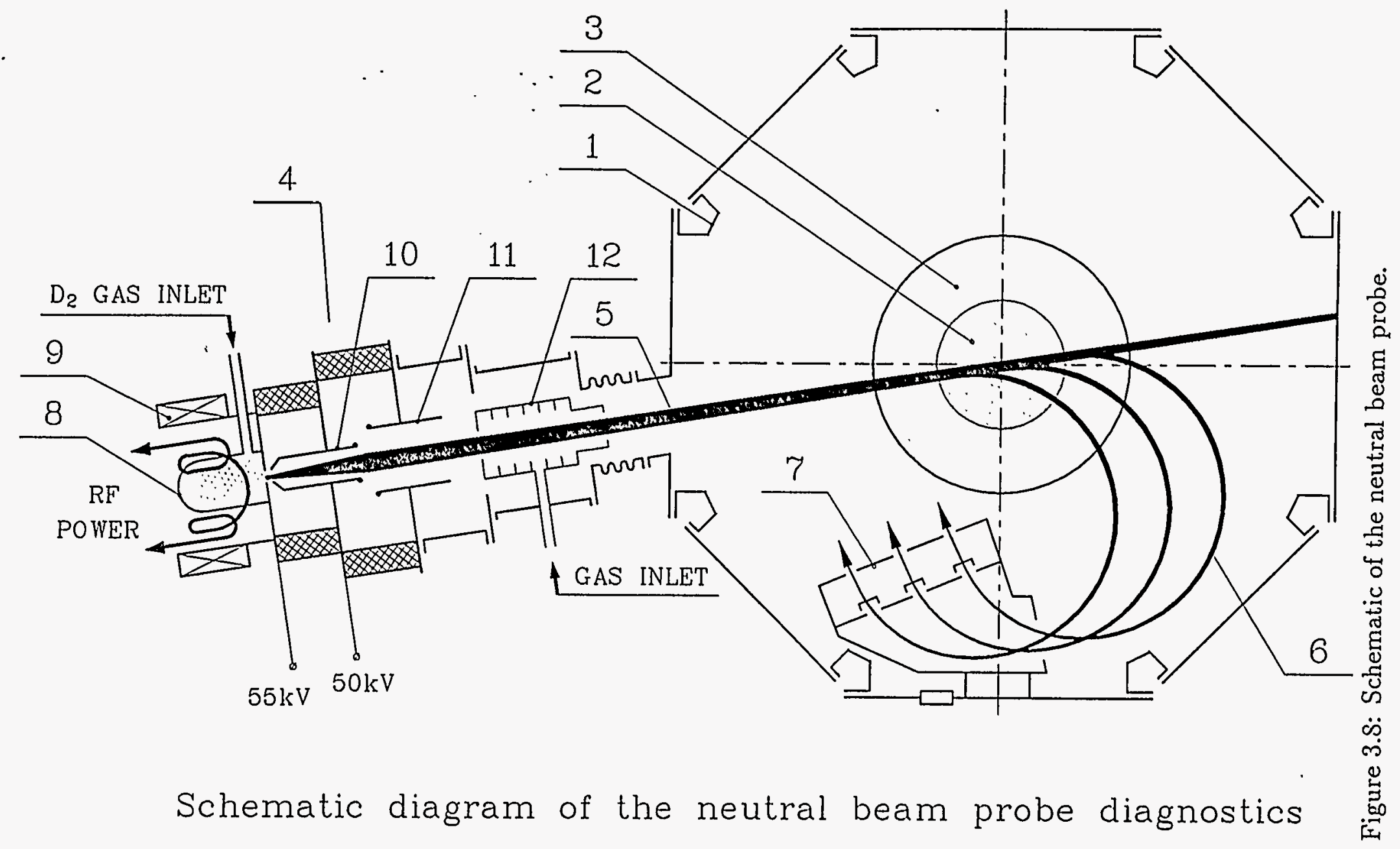

1-vacuum chamber ; 2-plasma cross section; 3-region occupied by sloshing ions; 4-ion source; 5 -deuterium beam; 6- secondery ion trajectories; 7-detectors ; 8-RF-discharge volume ; 9-magnetic coil ; 10-extracting electrode; 11-electrostatic lens ; 12-neutralization cell. 


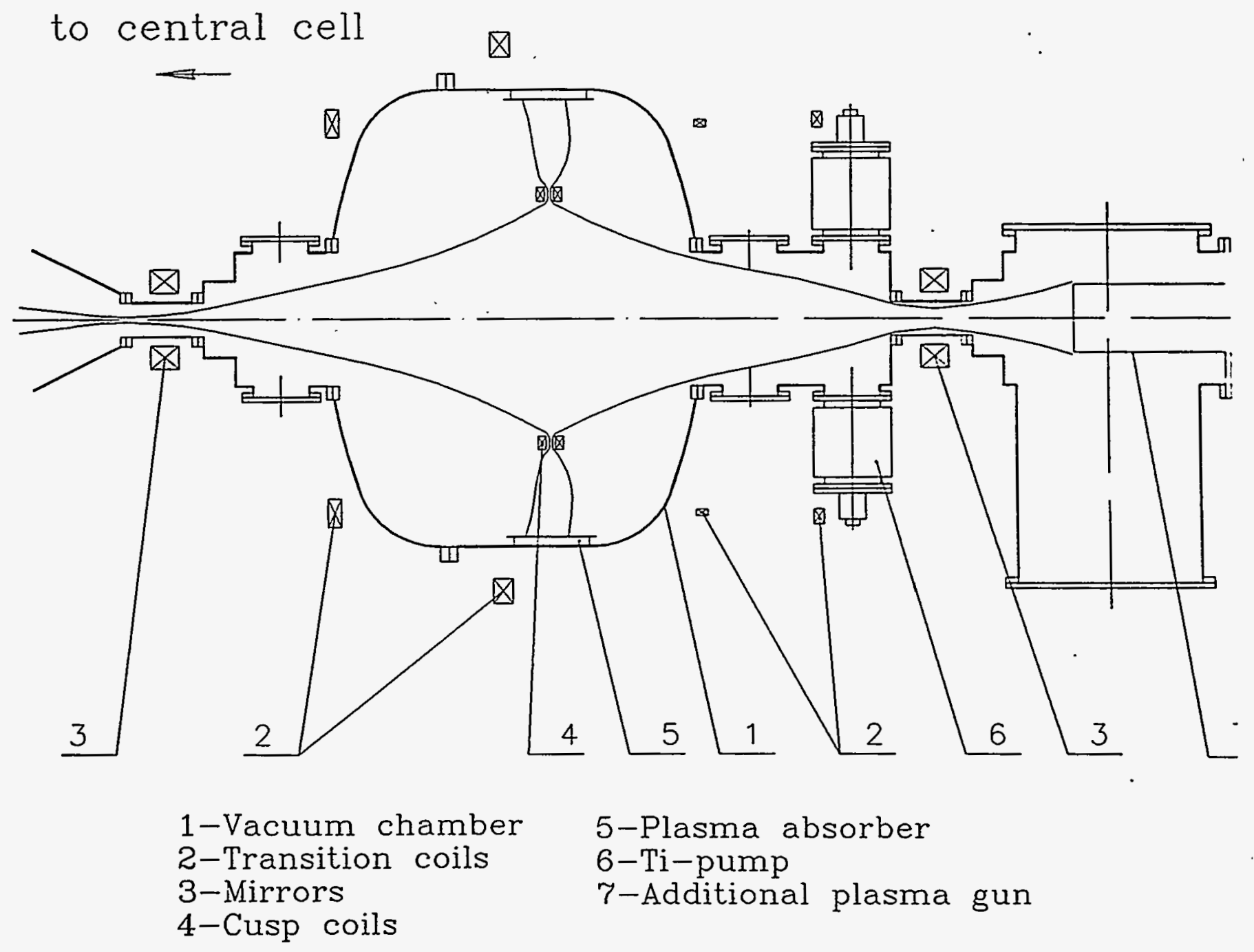

Figure 3.9: Schematic of Cusp End Cell. 


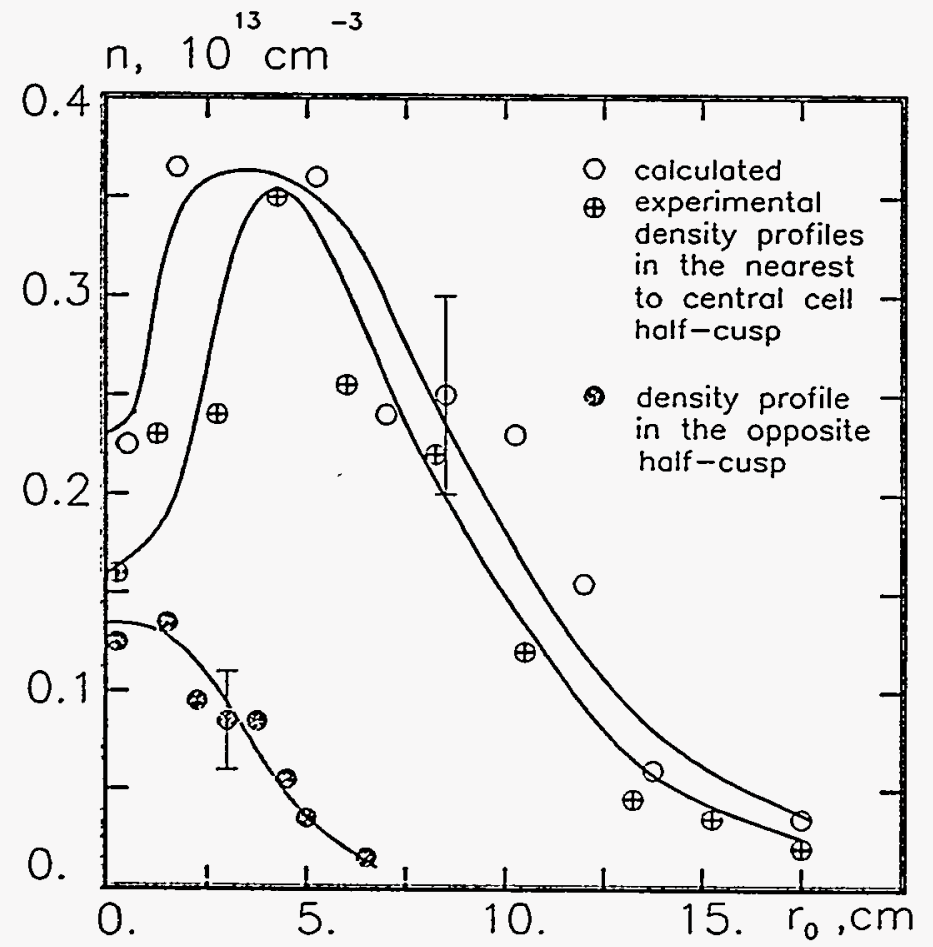

Figure 3.10: Plasma Profiles in the Cusp. 


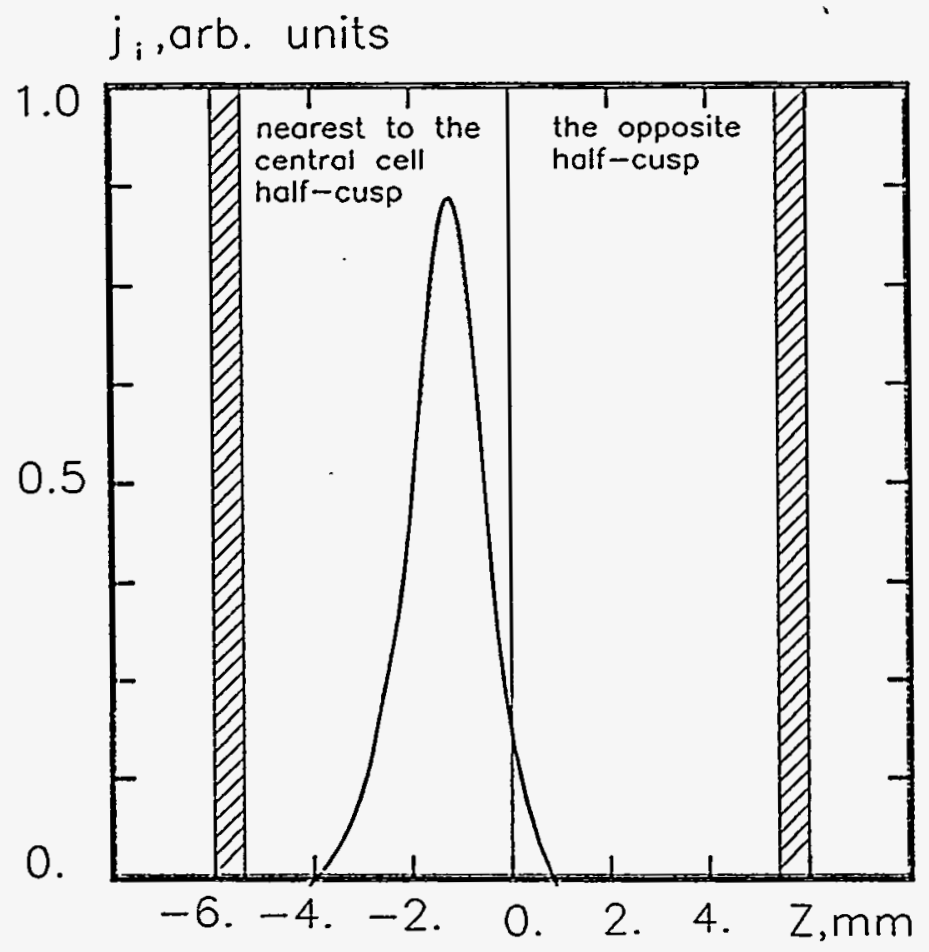

Figure 3.11: Plasma Profile in Ring Cusp. 


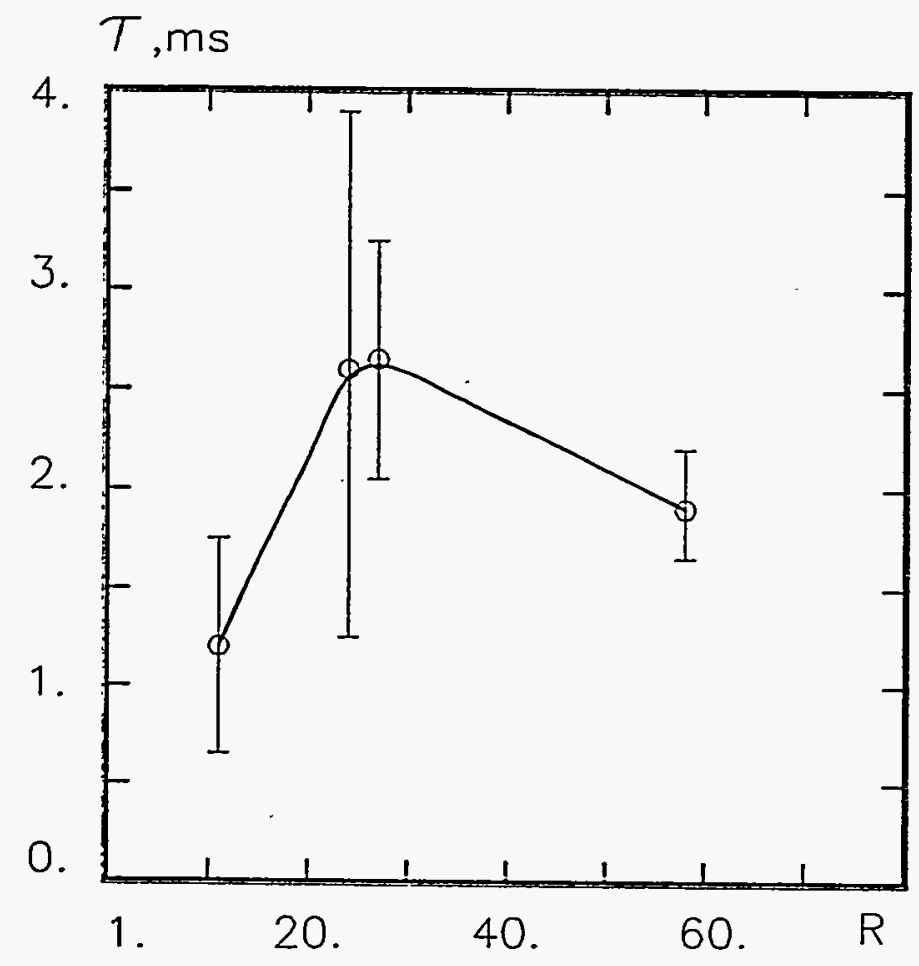

Figure 3.12: Plasma Lifetime as a Funtion of Mirror Ratio. 
$\mathrm{n}, 1 \mathrm{U} \mathrm{cm}$
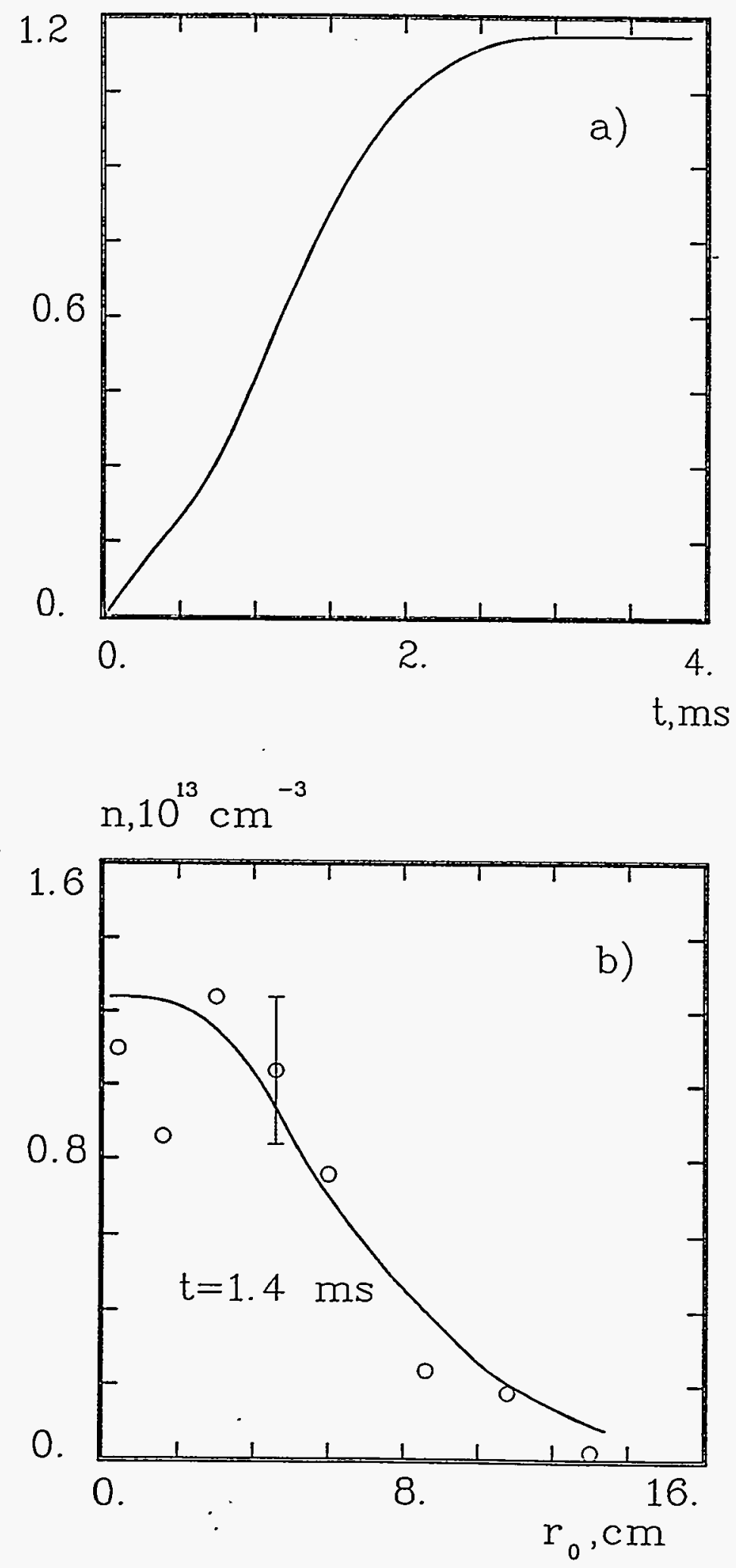

Figure 3.13: Plasma Parameters in the Cusp with additional Gun.

a.Temporal variation of on-axis Density;b. Plasma Profile in the Cusp. 


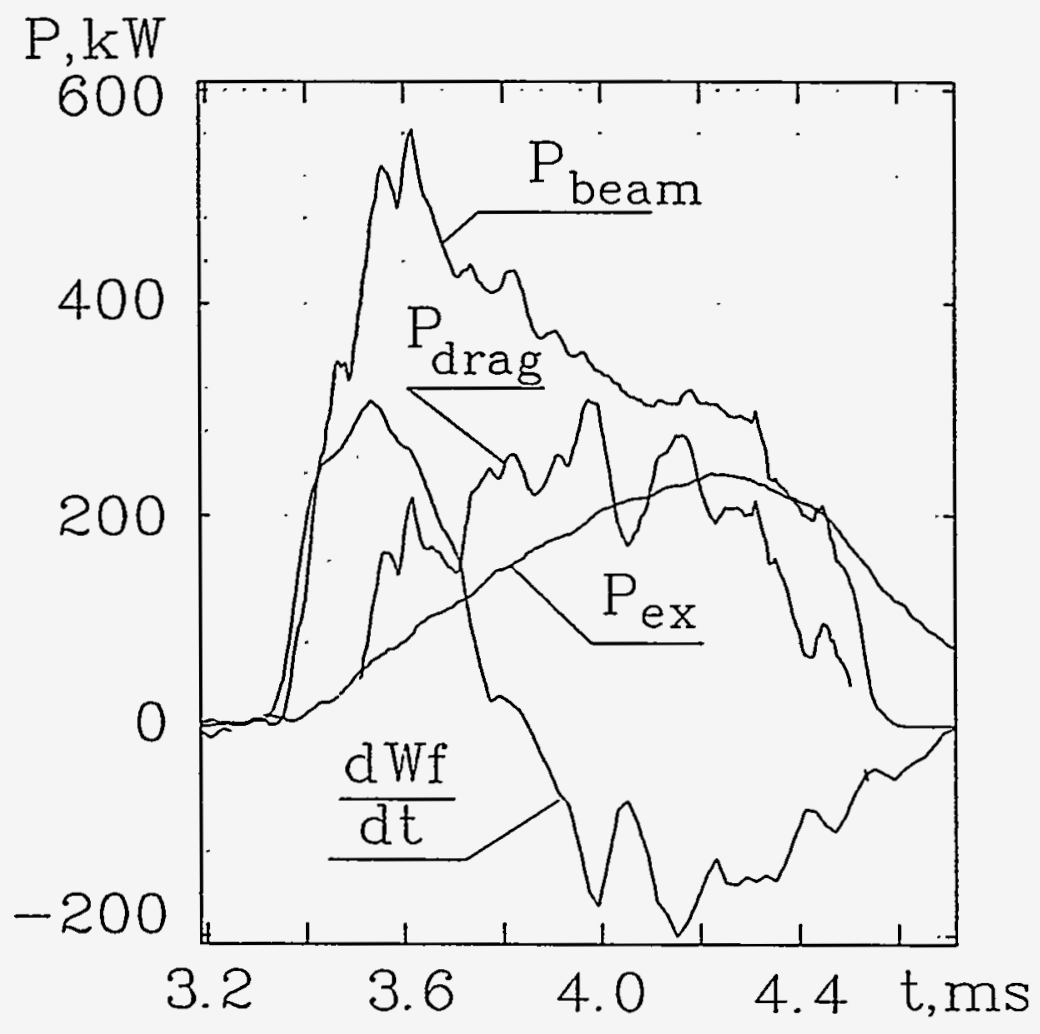

Figure 3.14: Energy balance during neutral beam heating.

1. $P_{\text {beam }}$-trapped power; 2. $P_{\text {drag }}$-power transmitted to plasma electrons; $3 . P_{e x}$-chargeexchange losses; 4 . $\frac{d W_{f}}{d t}$-growth rate of sloshing ion energy content. 


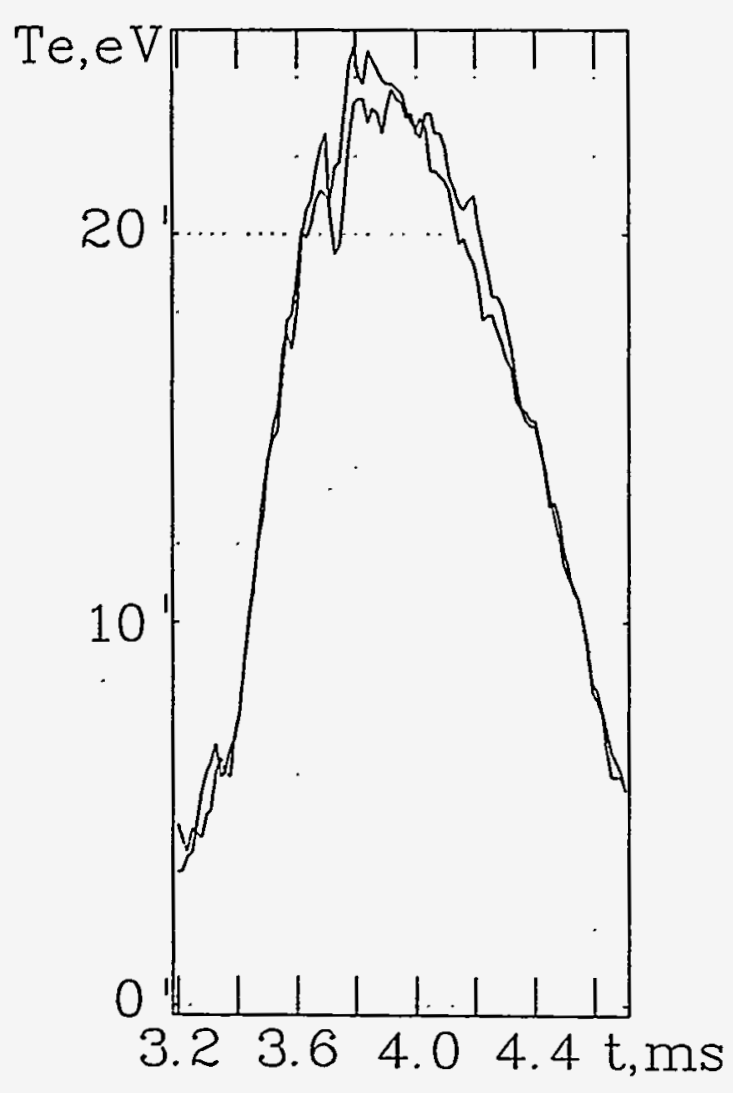

Figure 3.15: Electron temperature. 


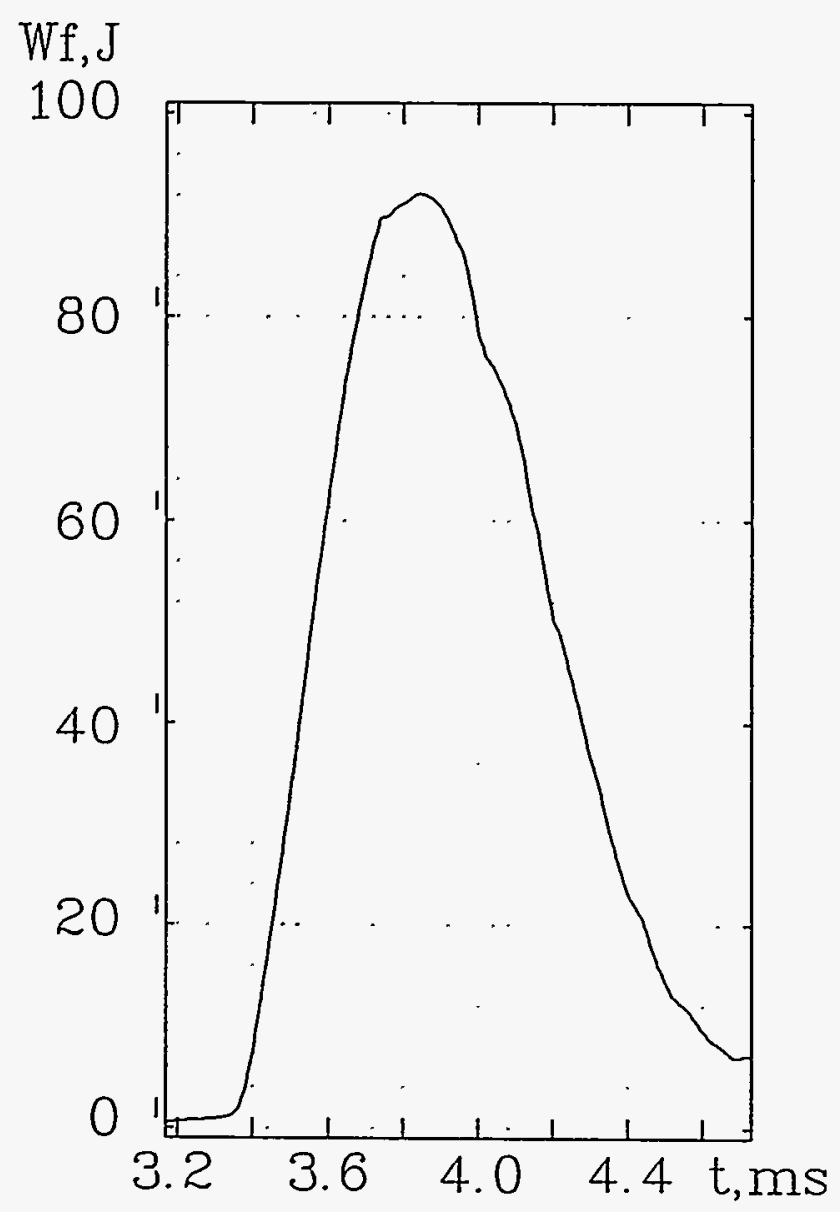

Figure 3.16: Energy Content of Sloshing Ions. 

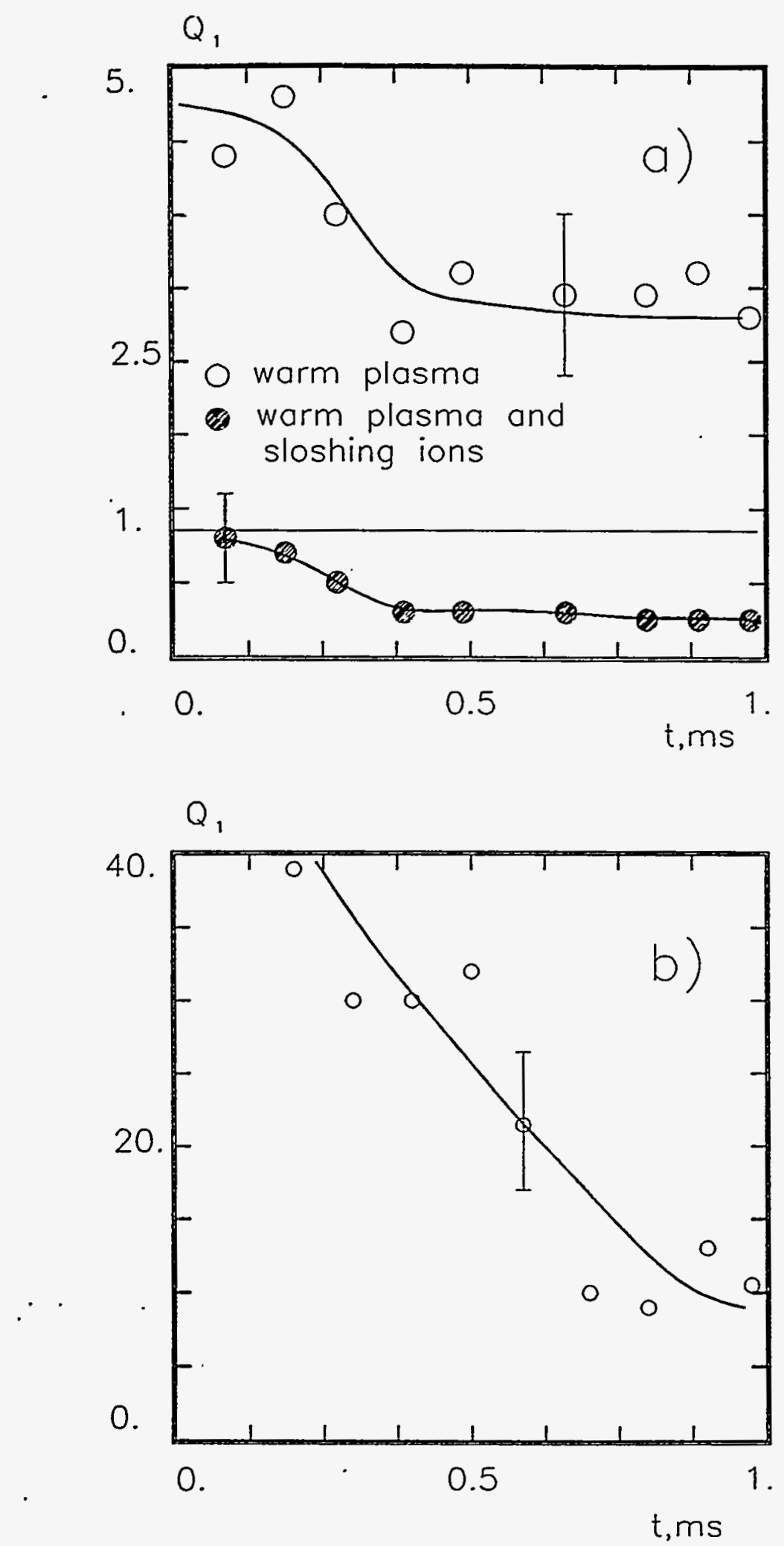

Figure 3.1S: Calculated Safety Factor During the Heating. a.For hollow profile in the Cusp.; b. With additional Gun On. 